\title{
The method of Thue-Siegel for binary quartic forms
}

\author{
by \\ Shabnam AkHtari (Bonn)
}

1. Introduction. In 1909, Thue [20] proved that if $F(x, y)$ is an irreducible binary form of degree at least 3 with integer coefficients, and $h$ a nonzero integer, then the equation $F(x, y)=h$ has only finitely many solutions in integers $x$ and $y$.

In this paper we will consider irreducible binary quartic forms with integer coefficients, i.e. polynomials of the shape

$$
F(x, y)=a_{0} x^{4}+a_{1} x^{3} y+a_{2} x^{2} y^{2}+a_{3} x y^{3}+a_{4} y^{4} .
$$

The discriminant $D$ of $F$ is given by

$D=D_{F}=a_{0}^{6}\left(\alpha_{1}-\alpha_{2}\right)^{2}\left(\alpha_{1}-\alpha_{3}\right)^{2}\left(\alpha_{1}-\alpha_{4}\right)^{2}\left(\alpha_{2}-\alpha_{3}\right)^{2}\left(\alpha_{2}-\alpha_{4}\right)^{2}\left(\alpha_{3}-\alpha_{4}\right)^{2}$, where $\alpha_{1}, \alpha_{2}, \alpha_{3}$ and $\alpha_{4}$ are the roots of

$$
F(x, 1)=a_{0} x^{4}+a_{1} x^{3}+a_{2} x^{2}+a_{3} x+a_{4} .
$$

Here, we will recall some well-known facts about the invariants of quartic forms. We refer the reader to 9 for more details. The invariants of $F$ form a ring, generated by two invariants of weights 4 and 6 , namely

$$
\begin{aligned}
& I=I_{F}=a_{2}^{2}-3 a_{1} a_{3}+12 a_{0} a_{4}, \\
& J=J_{F}=2 a_{2}^{3}-9 a_{1} a_{2} a_{3}+27 a_{1}^{2} a_{4}-72 a_{0} a_{2} a_{4}+27 a_{0} a_{3}^{2} .
\end{aligned}
$$

These are algebraically independent and every invariant is a polynomial in $I$ and $J$. For the invariant $D$, we have

$$
27 D=4 I^{3}-J^{2} \text {. }
$$

In what follows, we will just consider the forms $F$ for which the quantity $J_{F}$ is 0 , i.e. for which we have

$$
27 D=4 I^{3}
$$

2010 Mathematics Subject Classification: 11D25, 11D45.

Key words and phrases: quartic Thue equations, Thue-Siegel method, hypergeometric functions. 
Let $h$ be a positive integer. The number of solutions in integers $x$ and $y$ of the equation

$$
|F(x, y)|=h
$$

will be the focus of our study in this paper.

THEOREM 1.1. Let $F(x, y)$ be an irreducible binary quartic form with integer coefficients and positive discriminant that splits in $\mathbb{R}$. If $J_{F}=0$, then the Diophantine equation $|F(x, y)|=1$ possesses at most 12 solutions in integers $x$ and $y$ (with $(x, y)$ and $(-x,-y)$ regarded as the same).

In Section 11, we will summarize the result of our computations for binary forms with small discriminant. We will give some examples for quartic binary forms $F(x, y)$ satisfying the hypotheses of Theorem 1.1. where $|F(x, y)|=1$ has four or three solutions in integers $x$ and $y$. The author is not aware of any quartic binary form $F$ for which $|F(x, y)|=1$ has more than four solutions.

In 22 different methods are used to give an upper bound 61 upon the number of integral solutions to the equation $|F(x, y)|=1$, where $F$ is an irreducible binary quartic form with no restriction on the value of $J_{F}$ and with $\left|D_{F}\right|$ large enough. Moreover, it is shown in [2] that if the irreducible binary quartic form $F$ splits in $\mathbb{R}$ and has large discriminant, the Diophantine equation $|F(x, y)|=1$ has at most 36 solutions in integers $x$ and $y$.

ThEOREM 1.2. Let $F(x, y)$ be a reduced irreducible binary quartic form with integer coefficients and positive discriminant that splits in $\mathbb{R}$. If $J_{F}=0$, then the inequality $|F(x, y)| \leq h$ possesses at most 12 coprime solutions $(x, y)$ with $|y| \geq h^{3 / 4} /(3 I)^{1 / 8}$.

The definition of a reduced form is given in Section 3 . It turns out that each quartic binary form is equivalent to a reduced one (see [9]).

One reason for us to be interested in these results, despite quite serious restrictions upon $F$, is that we know important families of quartic forms with these properties. For example, a solution to the equation $a X^{4}-b Y^{2}=1$ gives rise to a solution to the Thue equation

$$
x^{4}+4 t x^{3} y-6 t x^{2} y^{2}-4 t^{2} x y^{3}+t^{2} y^{4}=t_{1}^{2},
$$

where $t_{1} \mid t$. We have applied the methods of this paper to treat the above Thue equation in [1].

The method of Thue and Siegel based on Padé approximation to binomial functions applies to broad families of binomial Thue equations, and both so-called "quantitative" results (see the works of Evertse [10, 12, for example) as well as effective results (via effective irrationality measures from Baker [4, 5] onwards) can be obtained from it. This method has also been used to study binary cubic forms with positive discriminant, for decades (see [11], 6]). In 1939, Krechmar [13] showed that when the discriminant 
of a quartic form $F(x, y)$ is sufficiently large $\left(D_{F} \gg h^{216 / 5}\right)$, the equation (1) has at most 20 solutions in integers $x$ and $y$, provided that $J_{F}=0$ and all roots of $F(x, 1)$ are real numbers. We will use a refinement of the Thue-Siegel method by Evertse [11] to obtain our results.

2. The method of Thue-Siegel. The main purpose of this section is to explain why we need the restriction $J_{F}=0$ in the statements of our theorems. The answer is hidden in the method we use, the method of ThueSiegel. The relationship between a system of approximations to an arbitrary cubic irrationality and Padé approximations to $\sqrt[3]{1-x}$ was first established by Thue [21. Siegel [15, 16] identified approximating polynomials in Thue's papers [21, 22] with hypergeometric polynomials and applied this method to bounding the number of solutions to Diophantine equations $f(x, y)=k$ for certain binary forms $f(x, y)$ of degree $r$. He also established bounds for the number of solutions to

$$
a x^{n}-b y^{n}=c,
$$

where $n \geq 3$ [17.

In this paper, we always suppose that $J_{F}=0$. In Section 5, we will show that if $J_{F}=0$ then there are linear forms $\xi=\xi(x, y)$ and $\eta=\eta(x, y)$ so that

$$
F(x, y)=\frac{1}{8 \sqrt{3 I A_{4}}}\left(\xi^{4}-\eta^{4}\right),
$$

where the quantity $A_{4}$ is defined in (7). We will use Padé approximation via hypergeometric polynomials to approximate $\eta / \xi$ with rational integers. The main idea here is to replace the construction of a family of dense approximations to $\eta / \xi$ by a family of rational approximations to the function $(1-z)^{1 / 4}$. Consider the system of linear forms $R_{r}(z)=-Q_{r}(z)+(1-z)^{1 / 4} P_{r}(z)$ that approximate $(1-z)^{1 / 4}$ at $z=0$, such that $R_{r}(z)=z^{2 r+1} \bar{R}_{r}(z), \bar{R}_{r}(z)$ is regular at $z=0$, and $P_{r}(z)$ and $Q_{r}(z)$ are polynomials of degree $r$. Thue [19, 21] explicitly found polynomials $P_{r}(z)$ and $Q_{r}(z)$ and Siegel [15] identified them in terms of hypergeometric polynomials. Refining the method of Siegel, Evertse [1] used the theory of hypergeometric functions to give an upper bound for the number of solutions to the equation $f(x, y)=1$, where $f$ is a cubic binary form with positive discriminant. Here we adjust Lemma 4 of [11] for quartic forms.

Lemma 2.1. Let $r, g$ be integers with $r \geq 1, g \in\{0,1\}$. Put

$$
\begin{aligned}
& A_{r, g}(z)=\sum_{m=0}^{r}\left(\begin{array}{c}
r-g+1 / 4 \\
m
\end{array}\right)\left(\begin{array}{c}
2 r-g-m \\
r-g
\end{array}\right)(-z)^{m}, \\
& B_{r, g}(z)=\sum_{m=0}^{r-g}\left(\begin{array}{c}
r-1 / 4 \\
m
\end{array}\right)\left(\begin{array}{c}
2 r-g-m \\
r
\end{array}\right)(-z)^{m} .
\end{aligned}
$$


(i) There exists a power series $F_{r, g}(z)$ such that for all complex numbers $z$ with $|z|<1$,

$$
A_{r, g}(z)-(1-z)^{1 / 4} B_{r, g}(z)=z^{2 r+1-g} F_{r, g}(z)
$$

and

$$
\left|F_{r, g}(z)\right| \leq \frac{\left(\begin{array}{c}
r-g+1 / 4 \\
r+1-g
\end{array}\right)\left(\begin{array}{c}
r-1 / 4 \\
r
\end{array}\right)}{\left(\begin{array}{c}
2 r+1-g \\
r
\end{array}\right)}(1-|z|)^{-\frac{1}{2}(2 r+1-g)} .
$$

(ii) For all complex numbers $z$ with $|1-z| \leq 1$ we have

$$
\left|A_{r, g}(z)\right| \leq\left(\begin{array}{c}
2 r-g \\
r
\end{array}\right) .
$$

(iii) For all complex numbers $z \neq 0$ and for $h \in\{1,0\}$ we have

$$
A_{r, 0}(z) B_{r+h, 1}(z) \neq A_{r+h, 1}(z) B_{r, 0}(z) .
$$

Proof. This lemma has been proven in [1].

3. Equivalent forms. We will call forms $F_{1}$ and $F_{2}$ equivalent if they are equivalent under the $S L_{2}(\mathbb{Z})$-action (i.e. if there exist integers $b, c, d$ and $e$ such that

$$
F_{1}(b x+c y, d x+e y)=F_{2}(x, y)
$$

for all $x$ and $y$, where $b e-c d= \pm 1$ ). Denote by $N_{F}$ the number of solutions in integers $x$ and $y$ of the Diophantine equation

$$
|F(x, y)|=h .
$$

Note that if $F_{1}$ and $F_{2}$ are equivalent, then $N_{F_{1}}=N_{F_{2}}, I_{F_{1}}=I_{F_{2}}$ and $J_{F_{1}}=J_{F_{2}}$.

Let us define, for a quartic form $F$, an associated quartic form, the Hessian $H$, by

$$
H(x, y)=\frac{d^{2} F}{d x^{2}} \frac{d^{2} F}{d y^{2}}-\left(\frac{d^{2} F}{d x d y}\right)^{2}
$$

Then

$$
H(x, y)=A_{0} x^{4}+A_{1} x^{3} y+A_{2} x^{2} y^{2}+A_{3} x y^{3}+A_{4} y^{4},
$$

where

$$
\begin{aligned}
& A_{0}=3\left(8 a_{0} a_{2}-3 a_{1}^{2}\right), \\
& A_{1}=12\left(6 a_{0} a_{3}-a_{1} a_{2}\right), \\
& A_{2}=6\left(3 a_{1} a_{3}+24 a_{0} a_{4}-2 a_{2}^{2}\right), \\
& A_{3}=12\left(6 a_{1} a_{4}-a_{2} a_{3}\right), \\
& A_{4}=3\left(8 a_{2} a_{4}-3 a_{3}^{2}\right) .
\end{aligned}
$$


We have the following identities (see Proposition 5 of [9]):

$$
\begin{aligned}
I_{H} & =12^{2} I_{F}^{2}, \\
J_{H} & =12^{3}\left(2 I_{F}^{3}-J_{F}^{2}\right)
\end{aligned}
$$

and

$$
D_{H}=12^{6} J_{F}^{2} D_{F},
$$

where $H$ is the Hessian of $F$, and $D_{F}, D_{H}$ are the discriminants of $F$ and $H$, respectively. From the identities in (7) and using algebraic manipulation, we have

$$
\begin{aligned}
& A_{0} A_{3}^{2}-A_{4} A_{1}^{2} \\
& \quad=12^{3}\left(a_{0} a_{3}^{2}-a_{4} a_{1}^{2}\right)\left(2 a_{2}^{3}-9 a_{1} a_{2} a_{3}+27 a_{1}^{2} a_{4}-72 a_{0} a_{2} a_{4}+27 a_{0} a_{3}^{2}\right) \\
& \quad=12^{3}\left(a_{0} a_{3}^{2}-a_{4} a_{1}^{2}\right) J_{F}
\end{aligned}
$$

and similarly,

$$
A_{3}^{3}+8 A_{1} A_{4}^{2}-4 A_{2} A_{3} A_{4}=12^{3}\left(a_{3}^{3}+8 a_{1} a_{4}^{2}-4 a_{2} a_{3} a_{4}\right) J_{F} .
$$

When $J_{F}=0$, we obtain

$$
\begin{aligned}
A_{0} A_{3}^{2} & =A_{4} A_{1}^{2}, \\
A_{3}^{3}+8 A_{1} A_{4}^{2} & =4 A_{2} A_{3} A_{4} .
\end{aligned}
$$

Therefore, when $A_{3} A_{4} \neq 0$,

$$
\begin{aligned}
H(x, y) & =A_{0} x^{4}+A_{1} x^{3} y+A_{2} x^{2} y^{2}+A_{3} x y^{3}+A_{4} y^{4} \\
& =\frac{1}{4 A_{3}^{2} A_{4}}\left(2 A_{1} A_{4} x^{2}+A_{3}^{2} x y+2 A_{4} A_{3} y^{2}\right)^{2} \\
& =\frac{1}{4 A_{3}^{2} A_{4}} W(x, y)^{2},
\end{aligned}
$$

where we define the quadratic form $W(x, y)=2 A_{1} A_{4} x^{2}+A_{3}^{2} x y+2 A_{4} A_{3} y^{2}$. So we get

$$
I_{H}=\left(\frac{A_{3}^{4}-16 A_{1} A_{4}^{2} A_{3}}{4 A_{3}^{2} A_{4}}\right)^{2} .
$$

From (8), we obtain

$$
\left|A_{3}^{4}-16 A_{1} A_{4}^{2} A_{3}\right|=\left|48 A_{3}^{2} A_{4} I_{F}\right| .
$$

In order to make good use of the above identities, we prove the following lemma:

Lemma 3.1. Let $F(x, y)$ be a quartic form with $J_{F}=0$. There exists a form equivalent to $F(x, y)$ for which $A_{3} A_{4} \neq 0$.

Proof. If $A_{4}=0$, then by 10 we have $A_{4}=A_{3}=0$, and therefore

$$
H(x, y)=x^{2}\left(A_{0} x^{2}+A_{1} x y+A_{2} y^{2}\right) \text {. }
$$


Let

$$
x=m X+l Y \quad \text { and } \quad y=p X+q Y,
$$

where $m, l, p$ and $q$ are integers satisfying $m q-l p= \pm 1$. Suppose that $\Phi_{1}(X, Y)$ is equivalent to $F(x, y)$ under this substitution with Hessian

$$
H_{\Phi_{1}}(X, Y)=A_{0}^{\prime} X^{4}+A_{1}^{\prime} X^{3} Y+A_{2}^{\prime} X^{2} Y^{2}+A_{3}^{\prime} X Y^{3}+A_{4}^{\prime} Y^{4} .
$$

We have

$$
A_{4}^{\prime}=H_{\Phi_{1}}(0,1)=H_{F}(l, q)=l^{2}\left(A_{0} l^{2}+A_{1} l q+A_{2} q^{2}\right) .
$$

If $H_{F}$ is identically zero then by (8) and (9), we will have $I_{F}=J_{F}=D_{F}=0$. But since we have assumed that $F(x, y)$ is irreducible, $H_{F}(x, y)$ is not identically zero. Therefore, the integers $l$ and $q$ can be chosen so that

$$
A_{4}^{\prime}=H_{F}(l, q) \neq 0 \text {. }
$$

Let $t \in \mathbb{Z}$ and put

$$
M=m+l t, \quad P=p+q t .
$$

Let $\Phi_{2}(X, Y)$ be the equivalent form to $F(x, y)$ under the substitution

$$
x=M X+l Y \text { and } y=P X+q Y,
$$

and $H_{\Phi_{2}}(X, Y)=A_{0}^{\prime \prime} X^{4}+A_{1}^{\prime \prime} X^{3} Y+A_{2}^{\prime \prime} X^{2} Y^{2}+A_{3}^{\prime \prime} X Y^{3}+A_{4}^{\prime \prime} Y^{4}$. Then replacing $x$ by $M X+l Y$ and $y$ by $P X+q Y$ in $H_{F}(x, y)$, we find that $A_{3}^{\prime \prime}$, the coefficient of the term $X Y^{3}$ in $H_{\Phi_{2}}(X, Y)$, is equal to

$$
\begin{aligned}
A_{3}^{\prime \prime}= & 4 M l^{3} A_{0}+\left(l^{3} P+3 M l^{2} q\right) A_{1}+\left(2 l^{2} P q+2 M l q^{2}\right) A_{2} \\
& +\left(q^{3} m+3 P q^{2} l\right) A_{3}+4 P q^{3} A_{4} \\
= & (m+l t)\left(4 l^{3} A_{0}+3 l^{2} q A_{1}+2 l q^{2} A_{2}+q^{3} A_{3}\right) \\
& +(p+q t)\left(l^{3} A_{1}+2 l^{2} q A_{2}+3 l q^{2} A_{3}+4 q^{3} A_{4}\right) \\
= & K+4 t\left(l^{4} A_{0}+l^{3} q A_{1}+l^{2} q^{2} A_{2}+l q^{3} A_{3}+q^{4} A_{4}\right) \\
= & K+4 t A_{4}^{\prime} .
\end{aligned}
$$

Since $A_{4}^{\prime} \neq 0$, the integer $t$ can be chosen so that $A_{3}^{\prime \prime} \neq 0$.

In the following, we will show that $F(x, y)$ or one of its equivalents (under the $G L_{2}(\mathbb{Z})$-action) satisfies

$$
\left|A_{4}\right|<4 I \text {. }
$$

From now on, we will suppose that $A_{3} A_{4} \neq 0$. Let

$$
x=m X+l Y \quad \text { and } \quad y=p X+q Y,
$$

where $m, l, p$ and $q$ are integers satisfying $m q-l p= \pm 1$. Let $\Phi(X, Y)$ be equivalent to $F(x, y)$ under this substitution and

$$
\Phi(X, Y)=a_{0}^{\prime} X^{4}+a_{1}^{\prime} X^{3} Y+a_{2}^{\prime} X^{2} Y^{2}+a_{3}^{\prime} X Y^{3}+a_{4}^{\prime} Y^{4} .
$$


We observe that

$$
A_{4}^{\prime}=H_{\Phi}(0,1)=H_{F}(l, q),
$$

where $H_{\Phi}(X, Y)=A_{0}^{\prime} X^{4}+A_{1}^{\prime} X^{3} Y+A_{2}^{\prime} X^{2} Y^{2}+A_{3}^{\prime} X Y^{3}+A_{4}^{\prime} Y^{4}$.

To continue, we will need the following proposition due to Hermite.

Proposition 3.2. Suppose that $f_{11} x^{2}+2 f_{12} x y+f_{22} y^{2}$ is a binary form with $D=f_{11} f_{22}-f_{12}^{2} \neq 0$. Then there is an integer pair $\left(u_{1}, u_{2}\right) \neq(0,0)$ for which

$$
0<\left|f_{11} u_{1}^{2}+2 f_{12} u_{1} u_{2}+f_{22} u^{2}\right|<\sqrt{\frac{4}{3}|D|} .
$$

Proof. See [7, p. 31].

Proposition 3.2 implies that we can choose $l$ and $q$ such that

$$
\begin{aligned}
0<\left|A_{4}^{\prime}\right| & =\frac{1}{\left|4 A_{3}^{2} A_{4}\right|}\left(2 A_{1} A_{4} l^{2}+A_{3}^{2} l q+2 A_{4} A_{3} q^{2}\right)^{2} \\
& <\frac{1}{\left|4 A_{3}^{2} A_{4}\right|}\left|\frac{1}{3}\left(A_{3}^{4}-16 A_{1} A_{4}^{2} A_{3}\right)\right|=4|I|,
\end{aligned}
$$

where the last equality comes from (11).

We have shown that the Hessian of $F$ satisfies

$$
\begin{aligned}
H(x, y) & =A_{0} x^{4}+A_{1} x^{3} y+A_{2} x^{2} y^{2}+A_{3} x y^{3}+A_{4} y^{4} \\
& =\frac{1}{4 A_{3}^{2} A_{4}}\left(2 A_{1} A_{4} x^{2}+A_{3}^{2} x y+2 A_{4} A_{3} y^{2}\right)^{2} .
\end{aligned}
$$

We will need some results due to Cremona [9]. Since we are using different notations in this paper, we will summarize Propositions 6 and 8 of 9 in Lemmas 3.3 and 3.4 . In particular, we note that the quartic polynomial $g_{4}(X)$ in [9] is equal to $\frac{-1}{3} H(x, 1)$ and its leading coefficient is equal to $-A_{0} / 3$.

Lemma 3.3. Suppose $F(x, y)$ is a quartic form with invariants $I$ and $J$ and Hessian $H(x, y)$. Let $\phi$ be a root of $X^{3}-3 I+J$. Then

$$
-\frac{1}{9} H(x, y)+\frac{4}{3} \phi F(x, y)=m(x, y)^{2},
$$

where $m(x, y)$ is a quadratic covariant of $F(x, y)$.

Proof. See Proposition 6(iv) of [9].

LEMma 3.4. Let $F(x, y)$ be a quartic form with real coefficients and leading coefficient $a_{0}$. Suppose that $F(x, 1)=0$ has four real roots. Order the roots $\phi_{i}$ of $X^{3}-3 I+J$ so that $4 a_{0} \phi_{1}>4 a_{0} \phi_{2}>4 a_{0} \phi_{3}$. Set $\phi=\phi_{2}$. Then $m(x, y)$ is a positive definite quadratic form with real coefficients, where $m(x, y)$ is the covariant of $F(x, y)$ defined in Lemma 3.3 .

Proof. This is Proposition 8(ii) of [9]. Note that the quantity $z$ in that proposition is equal to $-A_{0}$ and therefore is positive in our case. 
Following Definition 4 of [9], we say that the quartic form $F(x, y)=$ $a_{0} x^{4}+a_{1} x^{3} y+a_{2} x^{2} y^{2}+a_{3} x y^{3}+a_{4} y^{4}$ with positive discriminant is reduced if the positive definite quadratic form $m(x, y)$ is reduced. Here, we remark that the real quadratic form $f(x, y)=a x^{2}+b x y+c y^{2}$ is called reduced if

$$
|b| \leq a \leq c \text {. }
$$

Lemma 3.5. Let $F$ be the quartic form in Theorem 1.2 and $H$ be its Hessian. If $F$ is reduced then for integers $x, y$ we have

$$
|H(x, y)| \geq 36 I y^{4} \text {. }
$$

Proof. Suppose that $F(x, y)$ is reduced. Taking $\phi=0$ in Lemma 3.3 , we know that the algebraic covariant $\frac{-1}{9} H(x, y)$ is the square of a quadratic form, say

$$
\frac{-1}{9} H(x, y)=m(x, y)^{2} .
$$

We assume that $y \neq 0$. Put

$$
m(x, y)=y^{2} m(z)=y^{2}\left(A z^{2}+B z+C\right),
$$

where $z=x / y$. Note that $m(z)$ assumes a minimum equal to $\left(4 A C-B^{2}\right) / 4 A$ at $z=-B / 2 A$. Since

$$
m(x, y)^{2}=\frac{1}{36 A_{3}^{2} A_{4}}\left(2 A_{1} A_{4} x^{2}+A_{3}^{2} x y+2 A_{4} A_{3} y^{2}\right)^{2},
$$

by (11), we get

$$
4 A C-B^{2}=\frac{16 A_{1} A_{3} A_{4}^{2}-A_{3}^{4}}{-36 A_{3}^{2} A_{4}}= \pm \frac{4}{3} I .
$$

Recall that $A_{0}<0$ and hence, by (10), $A_{4}<0$. Since $I>0$ and $m(x, y)$ is reduced, we have $4 A C-B^{2}>0$ and

$$
A^{2} \leq A C \leq \frac{1}{3}\left(4 A C-B^{2}\right)=\frac{4}{9} I .
$$

Therefore, $m(x, y) \geq 2 \sqrt{I} y^{2}$.

So we can assume that $|H(x, y)| \geq h^{3} 12 \sqrt{3 I}$ when looking for pairs of solutions $(x, y)$ with $|y| \geq h^{3 / 4} /(3 I)^{1 / 8}$.

4. Reduction to a diagonal form. Our goal in this section will be to reduce the problem at hand to consideration of diagonal forms over a suitable imaginary quadratic field. The method of Thue-Siegel is particularly well suited for application to such forms. We will show

Lemma 4.1. Let $F$ be the binary form in Theorem 1.1. Then

$$
F(x, y)=\frac{1}{96 A_{3}^{2} A_{4} \sqrt{-3 I}}\left(\xi(x, y)^{4}-\eta(x, y)^{4}\right),
$$

where $\xi$ and $\eta$ are complex conjugate linear forms in $x$ and $y$. 
Proof. Let $H(x, y)=A_{0} x^{4}+A_{1} x^{3} y+A_{2} x^{2} y^{2}+A_{3} x y^{3}+A_{4} y^{4}$, with $A_{3} A_{4} \neq 0$, be the Hessian of $F(x, y)$. We can factor $2 A_{1} A_{4} x^{2}+A_{3}^{2} x y+$ $2 A_{4} A_{3} y^{2}$ over $\mathbb{C}$ as

$$
\xi(x, y) \eta(x, y)=2 A_{1} A_{4} x^{2}+A_{3}^{2} x y+2 A_{4} A_{3} y^{2},
$$

where $\xi$ and $\eta$ are linear forms. So we may write

$$
x=m \xi+l \eta, \quad y=p \xi+q \eta,
$$

for some $m, l, p, q \in \mathbb{C}$. Therefore,

$$
\begin{aligned}
F(x, y) & =F(m \xi+l \eta, p \xi+q \eta)=a_{0}^{\prime} \xi^{4}+a_{1}^{\prime} \xi^{3} \eta+a_{2}^{\prime} \xi^{2} \eta^{2}+a_{3}^{\prime} \xi \eta^{3}+a_{4}^{\prime} \eta^{4} \\
& =\Phi(\xi, \eta) .
\end{aligned}
$$

The Hessian $H^{\prime}(\xi, \eta)$ of $\Phi(\xi, \eta)$ satisfies

$$
\begin{aligned}
H^{\prime}(\xi, \eta) & =A_{0}^{\prime} \xi^{4}+A_{1}^{\prime} \xi^{3} \eta+A_{2}^{\prime} \xi^{2} \eta^{2}+A_{3}^{\prime} \xi \eta^{3}+A_{4}^{\prime} \eta^{4} \\
& =\Delta^{2} H(x, y)=\frac{\Delta^{2}}{4 A_{3}^{2} A_{4}} \xi^{2} \eta^{2} .
\end{aligned}
$$

Hence,

$$
A_{0}^{\prime}=A_{1}^{\prime}=A_{3}^{\prime}=A_{4}^{\prime}=0, \quad A_{2}^{\prime}=\Delta^{2} \frac{1}{4 A_{3}^{2} A_{4}} .
$$

On the other hand,

$$
A_{0}^{\prime}=3\left(8 a_{0}^{\prime} a_{2}^{\prime}-3 a_{1}^{\prime 2}\right), \quad A_{1}^{\prime}=12\left(6 a_{0}^{\prime} a_{3}^{\prime}-a_{1}^{\prime} a_{2}^{\prime}\right) .
$$

Using Maple, it is easy to check that for any form $F(x, y)$,

$$
-10 a_{4} A_{0}+2 a_{3} A_{1}-a_{2} A_{2}+a_{1} A_{3}-2 a_{0} A_{4}=6 J .
$$

So, for $\Phi(\xi, \eta)$, we obtain

$$
-10 a_{4}^{\prime} A_{0}^{\prime}+2 a_{3}^{\prime} A_{1}^{\prime}-a_{2}^{\prime} A_{2}^{\prime}+a_{1}^{\prime} A_{3}^{\prime}-2 a_{0}^{\prime} A_{4}^{\prime}=6 J_{\Phi}=6 \Delta^{4} J_{F}=0,
$$

where $a_{i}^{\prime}$ are the coefficients of $\Phi$ and $A_{i}^{\prime}$ are the coefficients of its Hessian. Therefore, by (14), $a_{2}^{\prime}=0$, and from the expressions for $A_{0}^{\prime}$ and $A_{4}^{\prime}$ respectively that result from (7), we have $a_{1}^{\prime}=a_{3}^{\prime}=0$, whereby

$$
F(x, y)=\Phi(\xi, \eta)=a_{0}^{\prime} \xi^{4}+a_{4}^{\prime} \eta^{4} .
$$

Observe that if

$$
2 A_{1} A_{4} x^{2}+A_{3}^{2} x y+2 A_{4} A_{3} y^{2}=(\alpha x+\beta y)(\gamma x+\delta y)
$$

then for any complex number $\lambda$, in (13) we may take $\xi=\lambda(\alpha x+\beta y)$ and $\eta=\mu(\gamma x+\delta y)$, where $\lambda \mu=1$. Our goal now is to determine the values of $\lambda$ and $\mu=1 / \lambda$ in $\xi=\lambda(\alpha x+\beta y)$ and $\eta=\mu(\gamma x+\delta y)$, so that $a_{4}^{\prime}=-a_{0}^{\prime}$. We have

$$
\left(\begin{array}{ll}
\lambda \alpha & \lambda \beta \\
\mu \gamma & \mu \delta
\end{array}\right)\left(\begin{array}{cc}
m & l \\
p & q
\end{array}\right)=\left(\begin{array}{ll}
1 & 0 \\
0 & 1
\end{array}\right)
$$


Thus,

$$
\left(\begin{array}{cc}
m & l \\
p & q
\end{array}\right)=\left(\begin{array}{cc}
\lambda \alpha & \lambda \beta \\
\mu \gamma & \mu \delta
\end{array}\right)^{-1}=\frac{1}{\lambda \mu(\alpha \delta-\beta \gamma)}\left(\begin{array}{cc}
\mu \delta & -\lambda \beta \\
-\mu \gamma & \lambda \alpha
\end{array}\right)
$$

whereby

$$
\frac{p}{q}=\frac{-\mu \gamma}{\lambda \alpha} \quad \text { and so } \quad q=-\frac{\lambda p \alpha}{\mu \gamma} .
$$

Since $\Phi(\xi, \eta)=a_{0}^{\prime} \xi^{4}+a_{4}^{\prime} \eta^{4}$, we have

$$
a_{0}^{\prime}=\Phi(1,0) \quad \text { and } \quad a_{4}^{\prime}=\Phi(0,1) .
$$

When $\eta=\mu(\gamma x+\delta y)=0$, we have $m=-\delta p / \gamma$, and when $\xi=\lambda(\alpha x+\beta y)=0$, we have $l=-\beta q / \alpha$. So we can write

$$
\begin{aligned}
& a_{0}^{\prime}=F(m, p)=F(-\delta p / \gamma, p)=\frac{p^{4}}{\gamma^{4}} F(-\delta, \gamma), \\
& a_{4}^{\prime}=F(l, q)=F(-\beta q / \alpha, q)=\frac{q^{4}}{\alpha^{4}} F(-\beta, \alpha) .
\end{aligned}
$$

Therefore, if we choose $\lambda$ and $\mu$ so that $\mu^{8}=\mu^{4} / \lambda^{4}=F(-\beta, \alpha) / F(\delta,-\gamma)$, then $-a_{0}^{\prime}=a_{4}^{\prime}$.

We have shown that $F(x, y)$ can be written as $a_{0}^{\prime}\left(\xi(x, y)^{4}-\eta(x, y)^{4}\right)$, where

$$
\xi=\lambda(\alpha x+\beta y), \quad \eta=\mu(\gamma x+\delta y)
$$

and $\lambda \mu=1$. It remains to calculate the value of $a_{0}^{\prime}$. Using (14) and (7), we get

$$
A_{2}^{\prime}=\Delta^{2} \frac{1}{4 A_{3}^{2} A_{4}}=6\left(3 a_{1}^{\prime} a_{3}^{\prime}+24 a_{0}^{\prime} a_{4}^{\prime}-2 a_{2}^{\prime}\right)=144 a_{0}^{\prime} a_{4}^{\prime} .
$$

Replacing $a_{4}^{\prime}$ by $-a_{0}^{\prime}$, we obtain

$$
a_{0}^{\prime 2}=-\frac{\Delta^{2}}{24^{2} A_{3}^{2} A_{4}},
$$

where $\Delta=m q-l p$ is the determinant of the matrix $\left(\begin{array}{cc}m & l \\ p & q\end{array}\right)$. Therefore, from (15) and the fact that $\lambda \mu=1$,

$$
a_{0}^{\prime 2}=-\frac{1}{(\alpha \delta-\beta \gamma)^{2} 24^{2} A_{3}^{2} A_{4}} .
$$

To calculate $(\alpha \delta-\beta \gamma)^{2}$, we recall that

$$
2 A_{1} A_{4} x^{2}+A_{3}^{2} x y+2 A_{3} A_{4} y^{2}=(\alpha x+\beta y)(\gamma x+\delta y) ;
$$

consequently, computing the discriminant of the above quadratic form and by (11),

$$
|\alpha \delta-\beta \gamma|^{2}=\left|A_{3}^{4}-16 A_{1} A_{4}^{2} A_{3}\right|=\left|48 A_{3}^{2} A_{4} I\right|,
$$


and therefore

$$
a_{0}^{\prime}= \pm \frac{1}{96 A_{3}^{2} A_{4} \sqrt{-3 I}}
$$

where $I=I_{F}$.

We will assume, without loss of generality, that

$$
a_{0}^{\prime}=\frac{1}{96 A_{3}^{2} A_{4} \sqrt{-3 I}}
$$

5. Resolvent forms. Suppose that $\xi$ and $\eta$ are linear forms in Lemma 4.1. Let us define

$$
\xi^{\prime}=\frac{\xi}{\left(12 A_{3}^{2}\right)^{1 / 4}\left|A_{4}\right|^{1 / 8}}, \quad \eta^{\prime}=\frac{\eta}{\left(12 A_{3}^{2}\right)^{1 / 4}\left|A_{4}\right|^{1 / 8}},
$$

so that

$$
F(x, y)=\frac{1}{8 \sqrt{3 I A_{4}}}\left(\xi^{\prime}(x, y)^{4}-\eta^{\prime}(x, y)^{4}\right) .
$$

Lemma 4.1 can be restated as follows:

Lemma 5.1. Let $F$ be the binary form in Theorem 1.1. Then

$$
F(x, y)=\frac{1}{8 \sqrt{3 I A_{4}}}\left(\xi(x, y)^{4}-\eta(x, y)^{4}\right),
$$

where $\xi$ and $\eta$ are complex conjugate linear forms in $x$ and $y$, with

$$
\xi^{4}, \eta^{4} \in \mathbb{Q}\left(\sqrt{A_{0} I / 3}\right) .
$$

Proof. For the binary form $F(x, y)$ with Hessian $H(x, y)$, the sextic covariant $Q(x, y)$ is defined by

$$
Q(x, y)=\frac{\delta F}{\delta x} \cdot \frac{\delta H}{\delta y}-\frac{\delta F}{\delta y} \cdot \frac{\delta H}{\delta x} .
$$

Since we have taken $H(x, y)=\frac{1}{4 A_{3}^{2} A_{4}}\left(2 A_{1} A_{4} x^{2}+A_{3}^{2} x y+2 A_{4} A_{3} y^{2}\right)^{2}$, we may write

$$
Q(x, y)=\frac{1}{2 A_{3}^{2} A_{4}} W(x, y) \psi(x, y)
$$

where

$$
\begin{aligned}
W(x, y) & =2 A_{1} A_{4} x^{2}+A_{3}^{2} x y+2 A_{4} A_{3} y^{2}, \\
\psi(x, y) & =\left(A_{3}^{2} x+4 A_{3} A_{4} y\right) \frac{\delta F}{\delta x}-\left(4 A_{1} A_{4} x+A_{3}^{2} y\right) \frac{\delta F}{\delta y} .
\end{aligned}
$$

We have (see equation (25) of [9])

$$
16 H^{3}+9 Q^{2}=4^{4} \cdot 3^{3} I H F^{2} .
$$

We remark that in [9], $g_{4}=\frac{-1}{3} H, g_{6}=\frac{-1}{36} Q$ and the invariants $I$ and $J$ are the negatives of our $I$ and $J$, respectively. Since $H(x, y)=\frac{1}{4 A_{3}^{2} A_{4}} W(x, y)^{2}$ 
is not identically zero, we can divide both sides of the above identity by $H(x, y)$ to get

$$
W(x, y)^{4}+9 A_{3}^{2} A_{4} \psi(x, y)^{2}=4^{4} \cdot 3^{3} I A_{3}^{4} A_{4}^{2} F(x, y)^{2} .
$$

Since $W(x, y)=\xi \eta$ and $F(x, y)=\frac{\xi^{4}-\eta^{4}}{96 A_{3}^{2} A_{4} \sqrt{-3 I}}, 20$ implies that

$$
\xi^{4} \eta^{4}+9 A_{3}^{2} A_{4} \psi(x, y)^{2}=\frac{1}{4}\left(-\xi^{8}-\eta^{8}+2 \xi^{4} \eta^{4}\right)
$$

and we obtain

$$
\left(\xi^{4}+\eta^{4}\right)^{2}=-36 A_{3}^{2} A_{4} \psi(x, y)^{2} .
$$

Therefore, by (10),

$$
\xi^{4}+\eta^{4}= \pm \frac{6 A_{3}^{2}}{A_{1}} \sqrt{-A_{0}} \psi(x, y) .
$$

Note that if all roots of $F(x, 1)$ are real then $I>0$ and $A_{0}<0$ (see [9, Proposition 7]). So we may write

$$
\xi^{4}+\eta^{4}=b \sqrt{-A_{0}},
$$

with $b \in \mathbb{Q}$. We have also seen that

$$
\xi^{4}-\eta^{4}=i a \sqrt{3 I}
$$

for some even integer $a$. Therefore, for integers $x, y$, the quantities $\xi(x, y)^{4}$ and $\eta(x, y)^{4}$ are complex conjugates and belong to $\mathbb{Q}\left(\sqrt{-A_{0}}, \sqrt{-3 I}\right)$. Moreover, $\sqrt{-A_{0}} \xi(x, y)^{4}$ and $\sqrt{-A_{0}} \eta(x, y)^{4}$ are algebraic integers in $\mathbb{Q}\left(\sqrt{A_{0} I / 3}\right)$. This is because

$$
\sqrt{-A_{0}}\left(\xi^{4}+\eta^{4}\right)= \pm \frac{-6 A_{0} A_{3}^{2}}{A_{1}} \psi(x, y),
$$

and by $10,, A_{1} \mid A_{0} A_{3}^{2}$. We will work in the number field $\mathbb{Q}\left(\sqrt{A_{0} I / 3}\right)$. We also have

$$
\frac{\xi^{4}}{\eta^{4}}=\frac{b \sqrt{-A_{0}}+i a \sqrt{3 I}}{b \sqrt{-A_{0}}-i a \sqrt{3 I}}=\frac{-A_{0} b^{2}-3 a^{2} I+i 6 a b \sqrt{-A_{0} I / 3}}{-A_{0} b^{2}+3 a^{2} I} .
$$

Therefore,

$$
\xi^{4} / \eta^{4} \in \mathbb{Q}\left(\sqrt{A_{0} I / 3}\right) .
$$

Note that, in (16), we started with two linear forms and continued with their fourth powers. Let the linear form $\xi=\xi(x, y)$ be a fourth root of $\xi(x, y)^{4}$ and define

$$
\eta(x, y)=\bar{\xi}(x, y)
$$

Indeed, $\eta(x, y)$ is a fourth root of $\eta^{4}$. Hence, when $F(x, 1)$ splits in $\mathbb{R}$, we can define the complex conjugate linear forms $\xi(x, y)$ and $\eta(x, y)$ so that

$$
\xi^{4}-\eta^{4}=96 A_{3}^{2} A_{4} \sqrt{-3 I} F(x, y)
$$


and

$$
|\xi \eta|=\left|2 A_{1} A_{4} x^{2}+A_{3}^{2} x y+2 A_{4} A_{3} y^{2}\right| .
$$

Now let us define

$$
\xi^{\prime}=\frac{\xi}{\left(12 A_{3}^{2}\right)^{1 / 4}\left|A_{4}\right|^{1 / 8}}, \quad \eta^{\prime}=\frac{\eta}{\left(12 A_{3}^{2}\right)^{1 / 4}\left|A_{4}\right|^{1 / 8}},
$$

so that

$$
F(x, y)=\frac{1}{8 \sqrt{3 I A_{4}}}\left(\xi^{\prime}(x, y)^{4}-\eta^{\prime}(x, y)^{4}\right) .
$$

From (7), for every pair of integers $(x, y)$, we have

$$
3 \mid \frac{1}{4 A_{3}^{2} A_{4}} W(x, y)^{2}=H(x, y) \text {. }
$$

This gives

$$
12 A_{3}^{2} A_{4} \mid W(x, y)^{2} .
$$

By (20), for every pair $(x, y)$ of integers, we have

$$
16 A_{3}^{2} A_{4} \mid \psi(x, y)^{2} .
$$

Using (21), we conclude that the real part of $\xi^{4}$ has the factor $12 A_{3}^{2} A_{4}$. Since $\xi^{4}-\eta^{4}=a_{0}^{\prime} F$, by 18 , the imaginary part of $\xi^{4}$ also has the factor $12 A_{3}^{2} A_{4}$. So

$$
\frac{\xi^{4}}{\left|12 A_{3}^{2} A_{4}\right|}, \frac{\eta^{4}}{\left|12 A_{3}^{2} A_{4}\right|} \in \mathbb{Q}\left(\sqrt{-A_{0}}, \sqrt{-3 I}\right) .
$$

By 10$)$,

$$
\frac{\sqrt{-A_{4}} \xi^{4}}{\left|12 A_{3}^{2} A_{4}\right|}, \frac{\sqrt{-A_{4}} \eta^{4}}{\left|12 A_{3}^{2} A_{4}\right|} \in \mathbb{Q}\left(\sqrt{A_{0} I / 3}\right) .
$$

We call a pair of complex conjugates $\xi$ and $\eta$ satisfying the identities in Lemma 5.1 a pair of resolvent forms, and note that if $(\xi, \eta)$ is one pair, there are precisely three others, given by $(i \xi,-i \eta),(-\xi,-\eta)$ and $(-i \xi, i \eta)$, where $i=\sqrt{-1}$. We will, however, work with $(\xi, \eta)$, a fixed pair of resolvent forms. For this pair, we have

$$
|\xi \eta|=\left|\frac{2 A_{1} A_{4} x^{2}+A_{3}^{2} x y+2 A_{4} A_{3} y^{2}}{\sqrt{12 A_{3}^{2} \sqrt{\left|A_{4}\right|}}}\right|=\frac{\left(H(x, y)^{2}\left|A_{4}\right|\right)^{1 / 4}}{\sqrt{3}} .
$$

REMARK. The fact that for integers $x$ and $y, \xi(x, y)^{4}$ and $\eta(x, y)^{4}$ are complex conjugates and belong to an imaginary quadratic field is crucial for our proof. To satisfy these conditions, when $J_{F}=0$, we only need $I_{F} A_{0}<0$ (see the proof of Lemma 5.1). Proposition 7 of [9] guarantees this property for quartic binary forms that split in $\mathbb{R}$. So we may generalize Theorem 1.1 to all quartic binary forms with $I_{F} A_{0}<0$. 
6. Gap principles. Let $\omega$ be a fourth root of unity (for some $j \in$ $\{1,2,3,4\}$, let $\left.\omega=e^{2 j \pi i / 4}\right)$. We say that the integer pair $(x, y)$ is related to $\omega$ if

$$
\left|\omega-\frac{\eta(x, y)}{\xi(x, y)}\right|=\min _{0 \leq k \leq 3}\left|e^{2 k \pi i / 4}-\frac{\eta(x, y)}{\xi(x, y)}\right| .
$$

Let us define $z=1-(\eta(x, y) / \xi(x, y))^{4}$, where $(\xi, \eta)$ is a fixed pair of resolvent forms (in other words, $\eta / \xi$ is a fourth root of $1-z$ ). We have

$$
|1-z|=1, \quad|z|<2 .
$$

Note that $|z|=2$ is impossible here, because it would mean $\eta^{4}=-\xi^{4}$, so $F(x, y)=\frac{1}{4 \sqrt{3 I A_{4}}} \xi^{4}$ and hence $D_{F}=0$.

LEMma 6.1. Let $\omega$ be a fourth root of unity and suppose the integral pair $(x, y)$ satisfies $F(x, y)=\frac{1}{8 \sqrt{3 I A_{4}}}\left(\xi(x, y)^{4}-\eta(x, y)^{4}\right)=1$ and is related to $\omega$. If $|z| \geq 1$ then

$$
\left|\omega-\frac{\eta(x, y)}{\xi(x, y)}\right| \leq \frac{\pi}{8}|z|
$$

If $|z|<1$ then

$$
\left|\omega-\frac{\eta(x, y)}{\xi(x, y)}\right|<\frac{\pi}{12}|z|
$$

Proof. Put

$$
4 \theta=\arg \left(\frac{\eta(x, y)^{4}}{\xi(x, y)^{4}}\right) .
$$

We have

$$
\sqrt{2-2 \cos (4 \theta)}=|z| \text {. }
$$

Therefore, when $|z|<2$ we have $|\theta|<\pi / 4$, and when $|z|<1$ we have $|\theta|<\pi / 12$. Since

$$
\left|\omega-\frac{\eta(x, y)}{\xi(x, y)}\right| \leq|\theta|
$$

we obtain

$$
\left|\omega-\frac{\eta(x, y)}{\xi(x, y)}\right| \leq \frac{1}{4} \frac{|4 \theta|}{\sqrt{2-2 \cos (4 \theta)}}\left|1-\frac{\eta(x, y)^{4}}{\xi(x, y)^{4}}\right| .
$$

By differential calculus $|4 \theta| / \sqrt{2-2 \cos (4 \theta)}<\pi / 2$ whenever $0<|\theta|<\pi / 4$. Therefore

$$
\left|\omega-\frac{\eta(x, y)}{\xi(x, y)}\right|<\frac{\pi}{8}|z|,
$$

and from the fact that $|4 \theta| / \sqrt{2-2 \cos (4 \theta)}<\pi / 3$ whenever $0<|\theta|<\pi / 12$, we conclude

as desired.

$$
\left|\omega-\frac{\eta(x, y)}{\xi(x, y)}\right|<\frac{\pi}{12}|z|
$$


Suppose that we have distinct solutions to $|F(x, y)| \leq h$ indexed by $i$, say $\left(x_{i}, y_{i}\right)$, related to a fixed fourth root of unity $\omega$ with $\left|\xi\left(x_{i+1}, y_{i+1}\right)\right| \geq$ $\left|\xi\left(x_{i}, y_{i}\right)\right|$. Let

$$
F\left(x_{i}, y_{i}\right)=h_{i}, \quad F\left(x_{i+1}, y_{i+1}\right)=h_{i+1} .
$$

For brevity, we will write $\eta_{i}=\eta\left(x_{i}, y_{i}\right)$ and $\xi_{i}=\xi\left(x_{i}, y_{i}\right)$. We have

$$
\left(\begin{array}{ll}
\lambda \alpha & \lambda \beta \\
\mu \gamma & \mu \delta
\end{array}\right)\left(\begin{array}{ll}
x_{1} & x_{2} \\
y_{1} & y_{2}
\end{array}\right)=\sqrt{12 A_{3}^{2} \sqrt{\left|A_{4}\right|}}\left(\begin{array}{ll}
\xi_{1} & \xi_{2} \\
\eta_{1} & \eta_{2}
\end{array}\right)
$$

(see the definition of the linear forms $\xi$ and $\eta$ in Section 5). Since $\left(x_{1}, y_{1}\right)$ and $\left(x_{2}, y_{2}\right)$ are distinct coprime solutions, $x_{1} y_{2}-x_{2} y_{1}$ is a nonzero integer. So by (17) and (10), we get

$$
\left|\xi_{1} \eta_{2}-\xi_{2} \eta_{1}\right|=\frac{\left|(\alpha \delta-\beta \gamma)\left(x_{1} y_{2}-x_{2} y_{1}\right)\right|}{\sqrt{12 A_{3}^{2} \sqrt{\left|A_{4}\right|}}} \geq 2 \sqrt{I}\left|A_{4}\right|^{1 / 4}
$$

On the other hand, by (23) and (24), we have

$$
\begin{aligned}
\left|\xi_{i} \eta_{i+1}-\xi_{i+1} \eta_{i}\right| & =\left|\xi_{i}\left(\eta_{i+1}-\omega \xi_{i+1}\right)-\xi_{i+1}\left(\eta_{i}-\omega \xi_{i}\right)\right| \\
& \leq\left|\xi_{i} \xi_{i+1}\left(\frac{\eta_{i+1}}{\xi_{i+1}}-\omega\right)\right|+\left|\xi_{i} \xi_{i+1}\left(\frac{\eta_{i}}{\xi_{i}}-\omega\right)\right| \\
& \leq \frac{\pi}{8}\left(\left|\xi_{i} \xi_{i+1} z_{i+1}\right|+\left|\xi_{i} \xi_{i+1} z_{i}\right|\right) \quad(\text { from the triangle inequality) } \\
& =\frac{\pi}{8}\left(\left|\xi_{i} \xi_{i+1} \frac{\eta_{i+1}^{4}-\xi_{i+1}^{4}}{\xi_{i+1}^{4}}\right|+\left|\xi_{i} \xi_{i+1} \frac{\eta_{i}^{4}-\xi_{i}^{4}}{\xi_{i}^{4}}\right|\right) \\
& \leq \pi h \sqrt{\left|3 I A_{4}\right|}\left(\frac{\left|\xi_{i}\right|}{\left|\xi_{i+1}^{3}\right|}+\frac{\left|\xi_{i+1}\right|}{\left|\xi_{i}^{3}\right|}\right),
\end{aligned}
$$

the last inequality holding from the expression for $F(x, y)$ in Lemma 5.1 and since $|F(x, y)|<h$. Since we assumed $\left|\xi_{i}\right| \leq\left|\xi_{i+1}\right|$, we get

$$
\left|\xi_{i} \eta_{i+1}-\xi_{i+1} \eta_{i}\right| \leq 2 \pi h \sqrt{\left|3 I A_{4}\right|} \frac{\left|\xi_{i+1}\right|}{\left|\xi_{i}^{3}\right|} .
$$

Combining this with 25), we conclude

$$
\left|\xi_{i+1}\right| \geq \frac{1}{\pi \sqrt{3} h\left|A_{4}\right|^{1 / 4}}\left|\xi_{i}\right|^{3} .
$$

Let us now assume that there are four distinct solutions to $|F(x, y)| \leq h$ related to a fixed choice of $\omega$, corresponding to $\xi_{-1}, \xi_{0}, \xi_{1}$ and $\xi_{2}$, where $\left|\xi_{-1}\right| \leq\left|\xi_{0}\right| \leq\left|\xi_{1}\right| \leq\left|\xi_{2}\right|$ and $F\left(x_{i}, y_{i}\right)=h_{i}$. We will deduce a contradiction, which shows that at most three such solutions can exist. By (26) and 
since $\left|h_{i}\right| \leq h$,

$$
\left|z_{i+1}\right| \leq \frac{3 \pi^{4}\left|z_{i}\right|^{3} h^{2}}{64 I}
$$

where $z_{i}=1-\eta_{i}^{4} / \xi_{i}^{4}=8 h \sqrt{\left|3 I A_{4}\right|} / \xi_{i}^{4}$. Since $\left|z_{-1}\right| \leq 2$, if $I>36.6 h^{2}$ then $\left|z_{0}\right|,\left|z_{1}\right|,\left|z_{2}\right|<1$. By (23),

$$
\begin{aligned}
\left|\xi_{-1} \eta_{0}-\xi_{0} \eta_{-1}\right| & =\left|\xi_{-1}\left(\omega \eta_{0}-\xi_{0}\right)-\xi_{0}\left(\omega \eta_{-1}-\xi_{-1}\right)\right| \\
& \leq 8 h\left(1+\frac{\pi}{12}\right) \sqrt{\left|3 I A_{4}\right|} \frac{\left|\xi_{0}\right|}{\left|\xi_{-1}^{3}\right|} .
\end{aligned}
$$

Combining this with (25), we conclude

$$
\left|\xi_{0}\right| \geq \frac{2 \sqrt{3}}{5 \pi h\left|A_{4}\right|^{1 / 4}}\left|\xi_{-1}\right|^{3} .
$$

Similarly, we get

$$
\begin{aligned}
\left|\xi_{0} \eta_{1}-\xi_{1} \eta_{0}\right| & =\left|\xi_{0}\left(\omega \eta_{1}-\xi_{1}\right)-\xi_{1}\left(\omega \eta_{0}-\xi_{0}\right)\right| \\
& \leq 8 h \sqrt{\left|3 I A_{4}\right|} \frac{\pi}{12}\left(\frac{\left|\xi_{0}\right|}{\left|\xi_{1}^{3}\right|}+\frac{\left|\xi_{1}\right|}{\left|\xi_{0}^{3}\right|}\right) \leq \frac{4 \pi}{3} h \sqrt{\left|3 I A_{4}\right|} \frac{\left|\xi_{1}\right|}{\left|\xi_{0}^{3}\right|}
\end{aligned}
$$

which leads to

$$
\left|\xi_{1}\right| \geq \frac{3}{2 \pi h\left|A_{4}\right|^{1 / 4}}\left|\xi_{0}\right|^{3} \geq \frac{72 \sqrt{3}}{2 \pi h^{4}(5 \pi)^{3}\left|A_{4}\right|}\left|\xi_{-1}\right|^{9} .
$$

Note that $\left|8 h \sqrt{\left|3 I A_{4}\right|} / \xi_{-1}^{4}\right|=\left|z_{-1}\right|=\left|1-\left(\eta_{-1} / \xi_{-1}\right)^{4}\right|<2$, and therefore

$$
\left|\xi_{-1}\right|^{4}>4 h \sqrt{\left|3 I A_{4}\right|} \text {. }
$$

Thus, when $I>36.6 h^{2}$ we have

$$
\left|\xi_{1}\right|>I^{9 / 8} \frac{72 \sqrt{3}(4 \sqrt{3})^{9 / 4}\left|A_{4}\right|^{1 / 8}}{2 \pi(5 \pi)^{3} h^{7 / 4}}>0.39 \frac{I^{9 / 8}\left|A_{4}\right|^{1 / 8}}{h^{7 / 4}} .
$$

Recall that by Lemma 3.5 , we can assume that $|H(x, y)| \geq h^{3} 12 \sqrt{3 I}$ when looking for pairs of solutions $(x, y)$ with $|y| \geq h^{3 / 4} /(3 I)^{1 / 8}$. This implies

$$
\left|H\left(x_{-1}, y_{-1}\right)\right| \geq 12 \frac{h^{3} \sqrt{3 I}}{\left|A_{3}^{2} A_{4}\right|}
$$

So by 22,

$$
\left|\xi_{-1}\right|^{4}=H \sqrt{\left|A_{4}\right|} / 3 \geq 4 h^{3} \sqrt{\left|3 I A_{4}\right|} .
$$

Moreover, one may assume that $h>2$, for the case $h=1$ has been addressed when we have been treating the Thue equation. Under these assumptions, we have

$$
\left|z_{-1}\right|=\left|\frac{8 h \sqrt{\left|3 I A_{4}\right|}}{\xi_{i}^{4}}\right|<1
$$


and by 27 and Lemma 6.1 .

$$
\left|\xi_{1}\right|>(4 \sqrt{3})^{9 / 4} I^{9 / 8} h^{11 / 4}\left|A_{4}\right|^{1 / 8}\left(\frac{3}{2 \pi}\right)^{4}>4 h^{11 / 4} I^{9 / 8}\left|A_{4}\right|^{1 / 8} .
$$

Here the point is that the inequality $|y| \geq h^{3 / 4} /(3 I)^{1 / 8}$ provides us with a good enough lower bound (30) for the size of $\xi_{1}$. Hence, to prove Theorem 1.2 , we do not need the assumption $I>36.6 h^{2}$.

7. Some algebraic numbers. Combining the polynomials $A_{r, g}$ and $B_{r, g}$ in Lemma 2.1 with the resolvent forms, we will consider the complex sequences $\Sigma_{r, g}$ given by

$$
\Sigma_{r, g}=\frac{\eta_{2}}{\xi_{2}} A_{r, g}\left(z_{1}\right)-\frac{\eta_{1}}{\xi_{1}} B_{r, g}\left(z_{1}\right)
$$

where $z_{1}=1-\eta_{1}^{4} / \xi_{1}^{4}$. For any pair of integers $(x, y), \xi(x, y)^{4}$ and $\eta(x, y)^{4}$ are algebraic integers in $\mathbb{Q}\left(\sqrt{A_{0} I / 3}\right)$ (see Lemma 5.1). We have seen that $A_{0}<0$ and one can assume $A_{3} A_{4} \neq 0$ (see Lemma 3.1). Therefore from (10), we have $A_{1} \neq 0$. Define

$$
\Lambda_{r, g}=\left(9\left|A_{4}\right|\right)^{(1-g) / 4} \xi_{1}^{4 r+1-g} \xi_{2} \Sigma_{r, g} .
$$

We will show that $\Lambda_{r, g}$ is either an integer in $\mathbb{Q}\left(\sqrt{A_{0} I / 3}\right)$ or a fourth root of such an integer. If $\Lambda_{r, g} \neq 0$, this provides a lower bound upon $\left|\Lambda_{r, g}\right|$.

Lemma 7.1. For any pair $(s, t)$ of integers, we have

$$
\frac{\xi(s, t)}{\xi(1,0)}, \frac{\eta(s, t)}{\eta(1,0)} \in \mathbb{Q}\left(\sqrt{A_{0} I / 3}\right)[s, t] .
$$

Proof. By (10) and (17), we have

$$
\alpha \delta-\beta \gamma=\sqrt{A_{3}^{4}-16 A_{1} A_{4}^{2} A_{3}}=\frac{4 A_{3}^{2}}{A_{1}} \sqrt{3 I A_{0}}=\frac{12 A_{3}^{2}}{A_{1}} \sqrt{\frac{I A_{0}}{3}} .
$$

Since

$$
\begin{aligned}
2 A_{1} A_{4} x^{2}+A_{3}^{2} x y+2 A_{3} A_{4} y^{2} & =(\alpha x+\beta y)(\gamma x+\delta y) \\
& =\sqrt{12 A_{3}^{2} \sqrt{\left|A_{4}\right|}} \xi(x, y) \eta(x, y),
\end{aligned}
$$

we conclude that $\alpha \gamma, \beta \delta, \alpha \delta+\beta \gamma \in \mathbb{Z}$. This implies the assertion.

Lemma 7.2. If $\left(x_{1}, y_{1}\right)$ and $\left(x_{2}, y_{2}\right)$ are two pairs of rational integers then

$$
\begin{gathered}
\sqrt{3\left|A_{4}\right|^{1 / 2}} \xi\left(x_{1}, y_{1}\right) \eta\left(x_{2}, y_{2}\right), \\
\xi\left(x_{1}, y_{1}\right)^{3} \xi\left(x_{2}, y_{2}\right)
\end{gathered}
$$

and

$$
\eta\left(x_{1}, y_{1}\right)^{3} \eta\left(x_{2}, y_{2}\right)
$$

are integers in $\mathbb{Q}\left(\sqrt{A_{0} I / 3}\right)$. 
Proof. For any pair of integers $(x, y)$, Lemma 7.1 implies that

$$
\frac{\xi(x, y)}{\xi(1,0)} \in \mathbb{Q}\left(\sqrt{A_{0} I / 3}\right) .
$$

Thus,

$$
\frac{\xi\left(x_{1}, y_{1}\right)}{\xi\left(x_{2}, y_{2}\right)} \in \mathbb{Q}\left(\sqrt{A_{0} I / 3}\right) .
$$

Since

$$
\sqrt{3\left|A_{4}\right|^{1 / 2}} \xi\left(x_{2}, y_{2}\right) \eta\left(x_{2}, y_{2}\right)=\frac{\omega(x, y)}{2\left|A_{3}\right|} \in \mathbb{Q},
$$

the algebraic integer $\sqrt{3\left|A_{4}\right|^{1 / 2}} \xi\left(x_{1}, y_{1}\right) \eta\left(x_{2}, y_{2}\right)$ belongs to $\mathbb{Q}\left(\sqrt{A_{0} I / 3}\right)$.

Let $\xi(x, y)=\epsilon_{1} x+\epsilon_{2} y$. Clearly, $\epsilon_{1}$ and $\epsilon_{2}$ are algebraic integers and so are $\epsilon_{1}^{4}, \epsilon_{1}^{3} \epsilon_{2}, \epsilon_{1}^{2} \epsilon_{2}^{2}, \epsilon_{1} \epsilon_{2}^{3}$ and $\epsilon_{2}^{4}$. Since $\xi^{4}$ is an integer in $\mathbb{Q}\left(\sqrt{A_{0} I / 3}\right)$, we conclude that $\epsilon_{1}^{4}, \epsilon_{1}^{3} \epsilon_{2}, \epsilon_{1}^{2} \epsilon_{2}^{2}, \epsilon_{1} \epsilon_{2}^{3}$ and $\epsilon_{2}^{4}$ are all algebraic integers in $\mathbb{Q}\left(\sqrt{A_{0} I / 3}\right)$.

Now, $\xi\left(x_{1}, y_{1}\right)^{3} \xi\left(x_{2}, y_{2}\right)$ is an integer in $\mathbb{Q}\left(\sqrt{A_{0} I / 3}\right)$, because it can be written as a linear combination with rational integer coefficients of $\epsilon_{1}^{4}, \epsilon_{1}^{3} \epsilon_{2}$, $\epsilon_{1}^{2} \epsilon_{2}^{2}, \epsilon_{1} \epsilon_{2}^{3}$ and $\epsilon_{2}^{4}$.

We can similarly show that $\eta\left(x_{1}, y_{1}\right)^{3} \eta\left(x_{2}, y_{2}\right)$ is also an integer in $\mathbb{Q}\left(\sqrt{A_{0} I / 3}\right)$.

For every polynomial $P(z)=a_{n} z^{n}+a_{n-1} z^{n-1}+\cdots+a_{1} z+a_{0}$, we define

$$
P^{*}(x, y)=x^{n} P(y / x)=a_{0} x^{n}+a_{1} x^{n-1} y+\cdots+a_{n-1} x y^{n-1}+a_{n} y^{n} .
$$

Let $A_{r, g}$ and $B_{r, g}$ be as in (2) and

$$
C_{r, g}(z)=A_{r, g}(1-z), \quad D_{r, g}(z)=B_{r, g}(1-z) .
$$

For $z \neq 0$, we have $D_{r, 0}(z)=z^{r} C_{r, 0}\left(z^{-1}\right)$, hence

$$
\begin{aligned}
A_{r, 0}^{*}(z, z-\bar{z}) & =z^{r} A_{r, 0}(1-\bar{z} / z)=z^{r} C_{r, 0}(\bar{z} / z) \\
& =\bar{z}^{r} D_{r, 0}(z / \bar{z})=\bar{z}^{r} B_{r, 0}(1-z / \bar{z}) \\
& =B_{r, 0}^{*}(\bar{z}, \bar{z}-z)=\bar{B}_{r, 0}^{*}(z, z-\bar{z}) .
\end{aligned}
$$

Lemma 7.3. For any pair $(x, y)$ of integers,

$$
A_{r, g}^{*}\left(\xi(x, y)^{4}, \xi(x, y)^{4}-\eta(x, y)^{4}\right) \quad \text { and } \quad B_{r, g}^{*}\left(\xi(x, y)^{4}, \xi(x, y)^{4}-\eta(x, y)^{4}\right)
$$

are algebraic integers in $\mathbb{Q}\left(\sqrt{A_{0} I / 3}\right)$.

Proof. It is clear that the above two elements belong to $\mathbb{Q}\left(\sqrt{A_{0} I / 3}\right)$. That they are algebraic integers follows immediately from Lemma 4.1 of [8] since

$$
\xi(x, y)^{4}-\eta(x, y)^{4}=8 h \sqrt{3 I A_{4}} F(x, y) .
$$


We now proceed to show that for any $r \in \mathbb{Z}, \Lambda_{r, 0}$ and $\Lambda_{r, 1}^{4}$ are integers in $\mathbb{Q}\left(\sqrt{A_{0} I / 3}\right)$. We have

$$
\begin{aligned}
\Lambda_{r, g} & =\left(9\left|A_{4}\right|\right)^{(1-g) / 4} \xi_{1}^{4 r} \xi_{1}^{1-g} \xi_{2} \Sigma_{r, g} \\
& =\left(9\left|A_{4}\right|\right)^{(1-g) / 4}\left(\xi_{1}^{1-g} \eta_{2} A_{r, g}^{*}\left(\xi_{1}^{4}, \xi_{1}^{4}-\eta_{1}^{4}\right)-\xi_{1}^{-g} \xi_{2} \eta_{1} B_{r, g}^{*}\left(\xi_{1}^{4}, \xi_{1}^{4}-\eta_{1}^{4}\right)\right) .
\end{aligned}
$$

For $g=0$, we obtain

$$
\Lambda_{r, 0}=\left(9\left|A_{4}\right|\right)^{1 / 4}\left(\xi_{1} \eta_{2} A_{r, 0}^{*}\left(\xi_{1}^{4}, \xi_{1}^{4}-\eta_{1}^{4}\right)-\xi_{2} \eta_{1} B_{r, 0}^{*}\left(\xi_{1}^{4}, \xi_{1}^{4}-\eta_{1}^{4}\right)\right) .
$$

By Lemma 7.2 , the numbers $\left(9\left|A_{4}\right|\right)^{1 / 4}\left(\xi_{1} \eta_{2}\right)$ and $\left(9\left|A_{4}\right|\right)^{1 / 4}\left(\xi_{2} \eta_{1}\right)$ are integers in $\mathbb{Q}\left(\sqrt{A_{0} I / 3}\right)$. They are also complex conjugates. From $\left.\sqrt{31}\right)$, Lemma 7.3 and the characterization of algebraic integers in quadratic number fields, we conclude that $\Lambda_{r, 0} \in \mathbb{Z} \sqrt{A_{0} I / 3}$. By Lemmas 7.2 and 7.3, $\Lambda_{r, 1}^{4}$ is an algebraic integer in $\mathbb{Q}\left(\sqrt{A_{0} I / 3}\right)$. Next we will show that $\Lambda_{r, 1}^{4}$ is not an integer when $\Sigma_{r, 1}$ is nonzero.

Suppose $\Lambda_{r, 1}^{4} \in \mathbb{Z}$. Then $\rho \Lambda_{r, 1}=\bar{\Lambda}_{r, 1}$ for some $\rho \in\{ \pm 1, \pm i\}$. Hence by the definition of $\Lambda_{r, 1}$ and since $\xi_{i}$ and $\eta_{i}$ are complex conjugates,

$$
\begin{aligned}
\rho \Sigma_{r, 1} & =\xi_{1}^{-4 r} \xi_{2}^{-1} \bar{\Lambda}_{r, 1} \\
& =\xi_{1}^{-4 r} \xi_{2}^{-1} \eta_{1}^{4 r} \eta_{2}\left(\frac{\xi_{2}}{\eta_{2}} A_{r, 1}\left(1-\frac{\xi_{1}^{4}}{\eta_{1}^{4}}\right)-\frac{\xi_{1}}{\eta_{1}} B_{r, 1}\left(1-\frac{\xi_{1}^{4}}{\eta_{1}^{4}}\right)\right) \\
& =\frac{\eta_{1}^{4 r}}{\xi_{1}^{4 r}}\left(A_{r, 1}\left(1-\frac{\xi_{1}^{4}}{\eta_{1}^{4}}\right)-\frac{\xi_{1} \eta_{2}}{\xi_{2} \eta_{1}} B_{r, 1}\left(1-\frac{\xi_{1}^{4}}{\eta_{1}^{4}}\right)\right) .
\end{aligned}
$$

This, together with Lemmas 7.2 and 7.3 , implies that

$$
\rho \Sigma_{r, 1} \in \mathbb{Q}\left(\sqrt{A_{0} I / 3}\right) .
$$

We have, by definition,

$$
\Sigma_{r, g}=\frac{\eta_{2}}{\xi_{2}} A_{r, g}\left(z_{1}\right)-\frac{\eta_{1}}{\xi_{1}} B_{r, g}\left(z_{1}\right)=\frac{\eta}{\xi}\left[\frac{\eta_{2} / \eta}{\xi_{2} / \xi} A_{r, g}\left(z_{1}\right)-\frac{\eta_{1} / \eta}{\xi_{1} / \xi} B_{r, g}\left(z_{1}\right)\right],
$$

where $\eta=\eta(1,0)$ and $\xi=\xi(1,0)$. By Lemmas 7.1 and 7.3 .

$$
\frac{\eta_{2} / \eta}{\xi_{2} / \xi} A_{r, g}\left(z_{1}\right)-\frac{\eta_{1} / \eta}{\xi_{1} / \xi} B_{r, g}\left(z_{1}\right) \in \mathbb{Q}\left(\sqrt{A_{0} I / 3}\right) .
$$

Hence

$$
\mathfrak{f}=\mathbb{Q}\left(\sqrt{A_{0} I / 3}, \rho \Sigma_{r, g}\right)=\mathbb{Q}\left(\sqrt{A_{0} I / 3}, \rho \xi / \eta\right) .
$$

If we choose a complex number $X$ so that $\xi(X, 1)=\eta(X, 1)$ then by Lemma 7.1. $X \in \mathfrak{f}$. We have $F(X, 1)=\frac{1}{8 \sqrt{3 I A_{4}}}\left(\xi(X, 1)^{4}-\eta(X, 1)^{4}\right)=0$. Since we have assumed that $F$ is irreducible, $X$ has degree 4 over $\mathbb{Q}$. But 
from $(32)$ and the definition of the number field $\mathfrak{f}$ in 33 ,

$$
X \in \mathfrak{f}=\mathbb{Q}\left(\sqrt{A_{0} I / 3}\right) .
$$

This contradicts the fact that $X$ has degree 4 over $\mathbb{Q}$. We conclude that $\Lambda_{r, 1}$ cannot be a rational integer.

From the well-known characterization of algebraic integers in quadratic fields, we may therefore conclude that if $\Lambda_{r, g} \neq 0$, then for $g \in\{0,1\}$,

$$
\left|\Lambda_{r, g}\right| \geq 2^{-g / 4}\left(-A_{0} I / 3\right)^{1 / 2-3 g / 8} .
$$

8. Approximating polynomials. In order to apply (25), we must make sure that $\Lambda_{r, g}$ or equivalently $\Sigma_{r, g}$ does not vanish. First we will show that for small $r, \Sigma_{r, 0} \neq 0$.

LEMMA 8.1. Suppose that $(x, y)$ is a pair of solutions to $F(x, y)= \pm 1$ with $I>135$ or a pair of solutions to $|F(x, y)| \leq h$ with $|y|>h^{3 / 4} /(3 I)^{1 / 8}$. For this pair of solutions and $r \in\{1,2,3,4,5\}$, we have

$$
\Sigma_{r, 0} \neq 0 \text {. }
$$

Proof. Let $r \in\{1,2,3,4,5\}$. Suppose that $\Sigma_{r, 0}=0$. From (3), we can find, for each $r$, a polynomial $F_{r}(z) \in \mathbb{Q}[z]$, satisfying

$$
A_{r, 0}(z)^{4}-(1-z) B_{r, 0}^{4}=z^{2 r+1} F_{r}(z) .
$$

In fact, using Maple, we have

$$
\begin{aligned}
A_{1}(z)= & 4 A_{1,0}(z)=8-5 z \\
B_{1}(z)= & 4 B_{1,0}(z)=8-3 z, \\
F_{1}(z)= & 320-320 z+81 z^{2} \\
A_{2}(z)= & \frac{32}{3} A_{2,0}(z)=64-72 z+15 z^{2}, \\
B_{2}(z)= & \frac{32}{3} B_{2,0}(z)=64-56 z+7 z^{2}, \\
F_{2}(z)= & 86016-172032 z+114624 z^{2}-28608 z^{3}+2401 z^{4}, \\
A_{3}(z)= & 128 A_{3,0}(z)=2560-4160 z+1872 z^{2}-195 z^{3}, \\
B_{3}(z)= & 128 B_{3,0}(z)=2560-3520 z+1232 z^{2}-77 z^{3}, \\
F_{3}(z)= & 14057472000-42172416000 z+48483635200 z^{2}-26679910400 z^{3} \\
& +7150266240 z^{4}-839047040 z^{5}+35153041 z^{6}, \\
A_{4}(z)= & \frac{2048}{5} A_{4,0}(z)=28672-60928 z+42432 z^{2}-10608 z^{3}+663 z^{4}, \\
B_{4}(z)= & \frac{2048}{5} B_{4,0}(z) \\
= & 28672-53760 z+31680 z^{2}-6160 z^{3}+231 z^{4}, \\
F_{4}(z)= & 13989396348928-55957585395712 z+91916125077504 z^{2} \\
& -79896826347520 z^{3}+39463764078592 z^{4}-11050000539648 z^{5} \\
& +1648475542656 z^{6}-113348764800 z^{7}+2847396321 z^{8}, \\
A_{5}(z)= & \frac{8192}{21} A_{5,0}(z) \\
= & 98304-258048 z+243712 z^{2}-99008 z^{3}+15912 z^{4}-663 z^{5},
\end{aligned}
$$




$$
\begin{aligned}
B_{5}(z)= & \frac{8192}{21} B_{5,0}(z) \\
= & 98304-233472 z+194560 z^{2}-66880 z^{3}+8360 z^{4}-209 z^{5}, \\
F_{5}(z)= & 121733331812352-608666659061760 z+1301756554248192 z^{2} \\
& -1555026262622208 z^{3}+1136607561252864 z^{4} \\
& -523630732640256 z^{5}+151029162176512 z^{6}-26204424888320 z^{7} \\
& +2515441608384 z^{8}-113971885760 z^{9}+1908029761 z^{10} .
\end{aligned}
$$

We also define

$$
A_{r}^{*}(x, y)=x^{r} A_{r}(y / x) \quad \text { and } \quad B_{r}^{*}(x, y)=x^{r} B_{r}(y / x) .
$$

Since $\Sigma_{r, 0}$ is assumed to be zero,

$$
\frac{\eta_{2}^{4}}{\xi_{2}^{4}}=\frac{\eta_{1}^{4}\left(B_{r}^{*}\left(\xi_{1}^{4}, \xi_{1}^{4}-\eta_{1}^{4}\right)\right)^{4}}{\xi_{1}^{4}\left(A_{r}^{*}\left(\xi_{1}^{4}, \xi_{1}^{4}-\eta_{1}^{4}\right)\right)^{4}} .
$$

Let $\mathfrak{I}_{r}$ be the integral ideal in $\mathbb{Q}\left(\sqrt{I A_{0} / 3}\right)$ generated by $\xi_{1}^{4}\left(A^{*}\left(\xi_{1}^{4}, \xi_{1}^{4}-\eta_{1}^{4}\right)\right)^{4}$ and $\eta_{1}^{4}\left(B^{*}\left(\xi_{1}^{4}, \xi_{1}^{4}-\eta_{1}^{4}\right)\right)^{4}$, and $N\left(\mathfrak{I}_{r}\right)$ be the absolute norm of $\mathfrak{I}_{r}$. Since the ideal generated by $\xi_{1}^{4}\left(A_{r}^{*}\left(\xi_{1}^{4}, \xi_{1}^{4}-\eta_{1}^{4}\right)\right)^{4}-\eta_{1}^{4}\left(B_{r}^{*}\left(\xi_{1}^{4}, \xi_{1}^{4}-\eta_{1}^{4}\right)\right)^{4}$ divides $\left(\xi_{2}^{4}-\eta_{2}^{4}\right) . \mathfrak{I}_{r}$, we obtain

$$
\begin{aligned}
\left|\xi_{1}\right|^{4(4 r+1)} \mid A_{r}^{4}\left(z_{1}\right)-(1 & \left.-z_{1}\right) B_{r}^{4}\left(z_{1}\right) \mid \\
& =\mid \xi_{1}^{4}\left(A_{r}^{*}\left(\xi_{1}^{4}, \xi_{1}^{4}-\eta_{1}^{4}\right)\right)^{4}-\eta_{1}^{4}\left(B_{r}^{*}\left(\xi_{1}^{4}, \xi_{1}^{4}-\eta_{1}^{4}\right)\right)^{4} .
\end{aligned}
$$

Since $\mathfrak{I}_{r}$ is an imaginary quadratic field, by $(18)$, we get

$$
\left|\xi_{1}\right|^{4(4 r+1)}\left|A_{r}^{4}\left(z_{1}\right)-\left(1-z_{1}\right) B_{r}^{4}\left(z_{1}\right)\right| \leq N\left(\mathfrak{I}_{r}\right)^{1 / 2}\left|\xi_{2}^{4}-\eta_{2}^{4}\right| .
$$

By (3),

$$
A_{r}^{4}\left(z_{1}\right)-\left(1-z_{1}\right) B_{r}^{4}\left(z_{1}\right)=z_{1}^{2 r+1} F_{r}\left(z_{1}\right),
$$

and so we conclude

$$
\left|z_{1}\right|^{2 r+1}\left|F_{r}\left(z_{1}\right)\right| \leq N\left(\mathfrak{I}_{r}\right)^{1 / 2}\left|\xi_{2}^{4}-\eta_{2}^{4}\right|\left|\xi_{1}\right|^{-4(4 r+1)},
$$

i.e.

$$
1 \leq \frac{N\left(\mathfrak{I}_{r}\right)^{1 / 2}\left|\xi_{2}^{4}-\eta_{2}^{4}\right|\left|\xi_{1}\right|^{-4(4 r+1)}}{\left|z_{1}\right|^{2 r+1}\left|F_{r}\left(z_{1}\right)\right|}
$$

Since $\xi_{1}^{4}=\left(\xi_{1}^{4}-\eta_{1}^{4}\right)\left(1-\eta_{1}^{4} / \xi_{1}^{4}\right)^{-1}=\left(\xi_{1}^{4}-\eta_{1}^{4}\right) z_{1}^{-1}$ we obtain

$$
1 \leq \frac{N\left(\mathfrak{I}_{r}\right)^{1 / 2}\left|\xi_{2}^{4}-\eta_{2}^{4}\right|\left|\xi_{1}^{4}-\eta_{1}^{4}\right|^{-4 r-1}\left|z_{1}\right|^{2 r}}{\left|F_{r}\left(z_{1}\right)\right|} .
$$

Noting that $\left|z_{1}\right|=\left|\xi_{1}^{-4}\right|\left|\xi_{1}^{4}-\eta_{1}^{4}\right|$ and $\left|\xi_{i}^{4}-\eta_{i}^{4}\right|=\left|8 h \sqrt{3 I A_{4}} F(x, y)\right|$, we obtain, for $r \in\{1,2,3,4,5\}$,

$$
\left|\xi_{1}\right|^{8 r} \leq \frac{\left(N\left(\mathfrak{I}_{r}\right)^{1 / 2}\left|\xi_{1}^{4}-\eta_{1}^{4}\right|^{-4 r-1}\right)\left|8 h \sqrt{3 I A_{4}}\right|^{2 r+1}}{\left|F_{r}\left(z_{1}\right)\right|} .
$$

To estimate $N\left(\mathfrak{I}_{r}\right)^{1 / 2}$, we choose a finite extension $\mathbf{M}$ of $\mathbb{Q}\left(\sqrt{A_{0} I / 3}\right)$ so that the ideal generated by $\xi_{1}^{4}$ and $\xi_{1}^{4}-\eta_{1}^{4}$ in $\mathbf{M}$ is a principal ideal, 
with generator $p$, say. We denote the extension of $\mathfrak{I}_{r}$ to $\mathbf{M}$ by $\mathfrak{I}_{r}^{\prime}$. Let $\mathfrak{r}_{r}$ be the ideal in $\mathbf{M}$ generated by $A_{r}^{*}(u, v)$ and $B_{r}^{*}(u, v)$, where $u=\xi_{1}^{4} / p$ and $v=\left(\xi_{1}^{4}-\eta_{1}^{4}\right) / p$. Since $A_{r}^{*}(x, x-y)=B_{r}^{*}(y, y-x)$,

$$
\begin{aligned}
p^{4 r+1} \mathfrak{r}_{r}^{4} B_{r}^{*}(0,1)^{4} & \subset p^{4 r+1} \mathfrak{r}_{r}^{4}\left(u, B_{r}^{*}(0, v)^{4}\right)\left(u-v, B_{r}^{*}(0, v)^{4}\right) \\
& \subset p^{4 r+1} \mathfrak{r}_{r}^{4}\left(u, B_{r}^{*}(0, v)^{4}\right)\left(u-v, A_{r}^{*}(v, v)^{4}\right) \\
& \subset p^{4 r+1} \mathfrak{r}_{r}^{4}(u, u-v)\left(u, B_{r}^{*}(u, v)^{4}\right)\left(u-v, A_{r}^{*}(u, v)^{4}\right) \\
& \subset p^{4 r+1}\left(u A^{*}(u, v)^{4},(u-v) B_{r}^{*}(u, v)^{4}\right)=\mathfrak{I}_{r}^{\prime},
\end{aligned}
$$

where $\left(m_{1}, \ldots, m_{n}\right)$ denotes the ideal in $\mathbf{M}$ generated by $m_{1}, \ldots, m_{n}$.

We have

$$
A_{1}^{*}(x, y)-B_{1}^{*}(x, y)=-2 y \text {. }
$$

Therefore,

$$
2(v) \subset\left(A_{1}^{*}(u, v), B_{1}^{*}(u, v)\right) \subset \mathfrak{r}_{1},
$$

where $(v)$ is the ideal generated by $v$ in $\mathbf{M}$. Since $B_{1}^{*}(0,1)=-3$, it follows from (36) that

$$
1296\left(\xi_{1}^{4}-\eta_{1}^{4}\right)^{5} \subset 1296 p\left(\xi_{1}^{4}-\eta_{1}^{4}\right)^{4}=p^{5} 16 v^{4} B_{1}^{*}(0,1)^{4} \subset \mathfrak{I}_{1}^{\prime} .
$$

For $r=2$, we first observe that

$$
B_{1}^{*}(x, y) A_{2}^{*}(x, y)-A_{1}^{*}(x, y) B_{2}^{*}(x, y)=-10 y^{3}
$$

and

$$
(-32 x+7 y) A_{2}^{*}(x, y)-(-32 x+15 y) B_{2}^{*}(x, y)=80 x y^{2} .
$$

Therefore, by (36) we have

$$
80(v)^{2} \subset\left(-10 v^{3}, 80 u v^{2}\right) \subset\left(A_{2}^{*}(u, v), B_{2}^{*}(u, v)\right) \subset \mathfrak{r}_{2} .
$$

Since $B_{2}^{*}(0,1)=7$, we have

$$
80^{4} \cdot 7^{4}\left(\xi_{1}^{4}-\eta_{1}^{4}\right)^{9} \subset 80^{4} \cdot 7^{4} p\left(\xi_{1}^{4}-\eta_{1}^{4}\right)^{8}=80^{4} p^{9} v^{8} B_{2}^{*}(0,1)^{4} \subset \mathfrak{I}_{2}^{\prime} .
$$

When $r=3$, we have

$$
\begin{gathered}
B_{2}^{*}(x, y) A_{3}^{*}(x, y)-A_{2}^{*}(x, y) B_{3}^{*}(x, y)=-210 y^{5}, \\
\left(1616 x^{2}-1078 x y+77 y^{2}\right) A_{3}^{*}(x, y)-\left(1616 x^{2}-1482 x y+195 y^{2}\right) B_{3}^{*}(x, y) \\
=-16800 x^{2} y^{3} .
\end{gathered}
$$

Substituting 77 for $B_{3}^{*}(0,1)$, we conclude

$$
\begin{aligned}
16800^{4} \cdot 77^{4}\left(\xi_{1}^{4}-\eta_{1}^{4}\right)^{13} & \subset 16800^{4} \cdot 77^{4} p\left(\xi_{1}^{4}-\eta_{1}^{4}\right)^{12} \\
& =16800^{4} p^{13} v^{12} B_{3}^{*}(0,1)^{4} \subset \mathfrak{I}_{3}^{\prime} .
\end{aligned}
$$

For $r=4$, setting

$$
\begin{aligned}
& G_{4}(x, y)=14178304 x^{3}-15889280 x^{2} y+4071760 x y^{2}-162393 y^{3}, \\
& H_{4}(x, y)=14178304 x^{3}-19433856 x^{2} y+6714864 x y^{2}-466089 y^{3},
\end{aligned}
$$


we may verify that

$$
\begin{aligned}
& B_{3}^{*}(x, y) A_{4}^{*}(x, y)-A_{3}^{*}(x, y) B_{4}^{*}(x, y)=-6006 y^{7}, \\
& G_{4}(x, y) A_{4}^{*}(x, y)-H_{4}(x, y) B_{4}^{*}(x, y)=-150678528 y^{4} x^{3} .
\end{aligned}
$$

These two identities imply that

$$
150678528^{4} \cdot 231^{4}\left(\xi_{1}^{4}-\eta_{1}^{4}\right)^{17} \subset 150678528^{4} \cdot 231^{4} p\left(\xi_{1}^{4}-\eta_{1}^{4}\right)^{16} .
$$

Since this last quantity is equal to $150678528^{4} p^{17} v^{16} B_{4}^{*}(0,1)^{4}$, from (36) it follows that

$$
150678528^{4} \cdot 231^{4}\left(\xi_{1}^{4}-\eta_{1}^{4}\right)^{17} \subset \mathfrak{I}_{4}^{\prime} .
$$

Finally, for $r=5$, set

$$
\begin{aligned}
G_{5}(x, y)= & 43706368 x^{4}-69346048 x^{3} y+32767856 x^{2} y^{2} \\
& -4764782 x y^{3}+123519 y^{4}, \\
H_{5}(x, y)= & 43706368 x^{4}-80272640 x^{3} y+46006896 x^{2} y^{2} \\
& -8845746 x y^{3}+391833 y^{4} .
\end{aligned}
$$

Then we have

$$
\begin{aligned}
& B_{4}^{*}(x, y) A_{5}^{*}(x, y)-A_{4}^{*}(x, y) B_{5}^{*}(x, y)=-14586 y^{7} \\
& G_{5}(x, y) A_{5}^{*}(x, y)-H_{5}(x, y) B_{5}^{*}(x, y)=-134424576 y^{5} x^{4} .
\end{aligned}
$$

These two identities imply that

$$
134424576^{4} \cdot 209^{4}\left(\xi_{1}^{4}-\eta_{1}^{4}\right)^{21} \subset 134424576^{4} \cdot 209^{4} p\left(\xi_{1}^{4}-\eta_{1}^{4}\right)^{20} .
$$

So by 36 ,

$$
134424576^{4} \cdot 209^{4}\left(\xi_{1}^{4}-\eta_{1}^{4}\right)^{21} \subset 134424576^{4} p^{21} v^{20} B_{5}^{*}(0,1)^{4} \subset \mathfrak{I}_{5}^{\prime} .
$$

From the preceding arguments, we are thus able to deduce the following series of inequalities:

$$
\begin{aligned}
& N\left(\mathfrak{I}_{1}\right)^{1 / 2}\left|\xi_{1}^{4}-\eta_{1}^{4}\right|^{-5} \leq 1296 \\
& N\left(\mathfrak{I}_{2}\right)^{1 / 2}\left|\xi_{1}^{4}-\eta_{1}^{4}\right|^{-9} \leq 560^{4} \\
& N\left(\mathfrak{I}_{3}\right)^{1 / 2}\left|\xi_{1}^{4}-\eta_{1}^{4}\right|^{-13} \leq(77 \cdot 16800)^{4} \\
& N\left(\mathfrak{I}_{4}\right)^{1 / 2}\left|\xi_{1}^{4}-\eta_{1}^{4}\right|^{-17} \leq(231 \cdot 150678528)^{4} \\
& N\left(\mathfrak{I}_{5}\right)^{1 / 2}\left|\xi_{1}^{4}-\eta_{1}^{4}\right|^{-21} \leq(134424576 \cdot 209)^{4} .
\end{aligned}
$$

Substituting any of these in (35) provides a contradiction to inequality (29) when $I>135$ and to 30 when $|y|>h^{3 / 4} /(3 I)^{1 / 8}$. Note that under both assumptions $I>135$ and $|y|>h^{3 / 4} /(3 I)^{1 / 8}$, the function $|z|=$ $\left|\xi^{-4}\right|\left|8 h \sqrt{3 I A_{4}} F(x, y)\right|$ is small. This makes $\left|F_{r}(z)\right|$ large enough for our contradictions.

LEMMA 8.2. If $r \in \mathbb{N}$ and $h \in\{0,1\}$, then at most one of $\left\{\Sigma_{r, 0}, \Sigma_{r+h, 1}\right\}$ can vanish. 
Proof. Let $r$ be a positive integer and $h \in\{0,1\}$. Following an argument of Bennett [6], we define the matrix $M$ :

$$
M=\left(\begin{array}{ccc}
A_{r, 0}\left(z_{1}\right) & A_{r+h, 1}\left(z_{1}\right) & \eta_{1} / \xi_{1} \\
A_{r, 0}\left(z_{1}\right) & A_{r+h, 1}\left(z_{1}\right) & \eta_{1} / \xi_{1} \\
B_{r, 0}\left(z_{1}\right) & B_{r+h, 1}\left(z_{1}\right) & \eta_{2} / \xi_{2}
\end{array}\right) .
$$

The determinant of $M$ is zero because it has two identical rows. Expanding along the first row, we get

$$
\begin{aligned}
0= & A_{r, 0}\left(z_{1}\right) \Sigma_{r+h, 1}-A_{r+h, 1}\left(z_{1}\right) \Sigma_{r, 0} \\
& +\frac{\eta_{2}}{\xi_{2}}\left(A_{r, 0}\left(z_{1}\right) B_{r+h, 1}\left(z_{1}\right)-A_{r+h, 1}\left(z_{1}\right) B_{r, 0}\left(z_{1}\right)\right) .
\end{aligned}
$$

If $\Sigma_{r, 0}=0$ and $\Sigma_{r+h, 1}=0$ then $A_{r, 0}\left(z_{1}\right) B_{r+h, 1}\left(z_{1}\right)-A_{r+h, 1}\left(z_{1}\right) B_{r, 0}\left(z_{1}\right)=0$, which contradicts Lemma 2.1(iii).

9. An auxiliary lemma. We now combine the lower bound for $\Lambda_{r, g}$ obtained in (34) with the upper bounds from Lemma 2.1 to prove the following lemma.

LEMma 9.1. If $\Sigma_{r, g} \neq 0$, then

$$
c_{1}(r, g)\left|\xi_{1}\right|^{4 r+1-g}\left|\xi_{2}\right|^{-3}+c_{2}(r, g)\left|\xi_{1}\right|^{-4 r-3(1-g)}\left|\xi_{2}\right|>1,
$$

where we may take

$$
\begin{aligned}
& c_{1}(1,0)=4 \pi h\left(\frac{3\left|A_{4}\right|^{3 / 2}}{\left|A_{0}\right|}\right)^{1 / 2}, \\
& c_{2}(1,0)=27 h^{3}\left(\frac{3\left|A_{4}\right|^{1 / 2}}{\left|A_{0}\right|}\right)^{1 / 2}\left(9 \sqrt{3 I\left|A_{4}\right|}\right)^{2} \frac{5}{128}
\end{aligned}
$$

and for $(r, g) \neq(1,0)$,

$$
\begin{aligned}
& c_{1}(r, g)=2 \sqrt{\pi} h\left(\frac{3\left|A_{4}\right|^{3 / 2}}{\left|A_{0}\right|}\right)^{1 / 2}\left(\frac{3\left|A_{4}\right|}{\left|A_{0}\right|^{3 / 2}}\right)^{-g / 4} \frac{4^{r}}{\sqrt{r}}, \\
& c_{2}(r, g)=27 h^{2 r+1-g}\left(\frac{3\left|A_{4}\right|^{1 / 2}}{\left|A_{0}\right|}\right)^{1 / 2}\left(\frac{3\left|A_{4}\right|}{\left|A_{0}\right|^{3 / 2}}\right)^{-g / 4}\left(9 \sqrt{3 I\left|A_{4}\right|}\right)^{2 r-g} \frac{\sqrt{2}}{\sqrt{r} \pi 4^{r}} .
\end{aligned}
$$

Proof. By the definition of $\Lambda_{r, g}$ and (3), we can write $\left|\Lambda_{r, g}\right|=\left(9\left|A_{4}\right|\right)^{(1-g) / 4}\left|\xi_{1}\right|^{4 r+1-g}\left|\xi_{2}\right|\left|\left(\eta_{2} / \xi_{2}-\omega\right) A_{r, g}\left(z_{1}\right)+\omega z_{1}^{2 r+1-g} F_{r, g}\left(z_{1}\right)\right|$. Since $\left|1-z_{1}\right|=1,\left|z_{1}\right| \leq 1$ and $\left|z_{i}\right|=8 h \sqrt{3 I} /\left|\xi_{i}^{4}\right|$, by (4), (5) and inequality (24), we have

$$
\left|\Lambda_{r, g}\right| \leq\left(9\left|A_{4}\right|\right)^{(1-g) / 4}\left|\xi_{1}\right|^{4 r+1-g}\left|\xi_{2}\right| \mathfrak{L},
$$


where

$$
\mathfrak{L}=\left(\begin{array}{c}
2 r-g \\
r
\end{array}\right) \frac{2 \pi h \sqrt{3 I\left|A_{4}\right|}}{3\left|\xi_{2}^{4}\right|}+\frac{\left(\begin{array}{c}
r-g+1 / 4 \\
r+1-g
\end{array}\right)\left(\begin{array}{c}
r-1 / 4 \\
r
\end{array}\right)}{\left(\begin{array}{c}
2 r+1-g \\
r
\end{array}\right)}\left(\frac{9 h \sqrt{3 I\left|A_{4}\right|}}{\left|\xi_{1}^{4}\right|}\right)^{2 r+1-g} .
$$

Comparing this with (34), we obtain

$$
c_{1}(r, g)\left|\xi_{1}\right|^{4 r+1-g}\left|\xi_{2}\right|^{-3}+c_{2}(r, g)\left|\xi_{1}\right|^{-4 r-3(1-g)}\left|\xi_{2}\right|>1,
$$

where we may take $c_{1}$ and $c_{2}$ so that

$$
c_{1}(r, g) \geq 2 \pi h\left(\frac{3\left|A_{4}\right|^{3 / 2}}{\left|A_{0}\right|}\right)^{1 / 2}\left(\frac{3\left|A_{4}\right|}{\left|A_{0}\right|^{3 / 2}}\right)^{-g / 4}\left(\begin{array}{c}
2 r \\
r
\end{array}\right)
$$

and

$c_{2}(r, g)$

$$
\geq 27 h^{2 r+1-g}\left(\frac{3\left|A_{4}\right|^{1 / 2}}{\left|A_{0}\right|}\right)^{1 / 2}\left(\frac{3\left|A_{4}\right|}{\left|A_{0}\right|^{3 / 2}}\right)^{-g / 4}\left(9 \sqrt{3 I\left|A_{4}\right|}\right)^{2 r-g} \frac{\left(\begin{array}{c}
r-g+1 / 4 \\
r+1-g
\end{array}\right)\left(\begin{array}{c}
r-1 / 4 \\
r
\end{array}\right)}{\left(\begin{array}{c}
2 r+1-g \\
r
\end{array}\right)} .
$$

Substituting $r=1$ and $g=0$, we get the desired values for $c_{1}(1,0)$ and $c_{2}(1,0)$. Let us apply the following version of Stirling's formula (see Theorem (5.44) of [18]):

$$
\frac{1}{2 \sqrt{k}} 4^{k} \leq\left(\begin{array}{c}
2 k \\
k
\end{array}\right)<\frac{1}{\sqrt{\pi k}} 4^{k}
$$

for $k \in \mathbb{N}$. This leads to the stated choice of $c_{1}$ immediately.

To evaluate $c_{2}(r, g)$, we first note that

$$
\left(\begin{array}{c}
2 r+1-g \\
r
\end{array}\right) \geq\left(\begin{array}{c}
2 r \\
r
\end{array}\right) \geq \frac{4^{r}}{2 \sqrt{r}}
$$

Next we will show that

$$
\left(\begin{array}{c}
r-g+1 / 4 \\
r+1-g
\end{array}\right)\left(\begin{array}{c}
r-1 / 4 \\
r
\end{array}\right)<\frac{1}{\sqrt{2} \pi r}
$$

for $r \in \mathbb{N}$ and $g \in\{0,1\}$, whence we may conclude that

$$
\frac{\left(\begin{array}{c}
r-g+1 / 4 \\
r+1-g
\end{array}\right)\left(\begin{array}{c}
r-1 / 4 \\
r
\end{array}\right)}{\left(\begin{array}{c}
2 r+1-g \\
r
\end{array}\right)}<\frac{\sqrt{2}}{\sqrt{r} \pi 4^{r}} .
$$

This leads immediately to the stated choice of $c_{2}$. It remains to show 38 .

Let us set

$$
X_{r}=\left(\begin{array}{c}
r-3 / 4 \\
r
\end{array}\right)\left(\begin{array}{c}
r-1 / 4 \\
r
\end{array}\right)=\frac{y_{r}}{r}
$$

whereby

$$
X_{r+1}=\left(\begin{array}{c}
r+1 / 4 \\
r+1
\end{array}\right)\left(\begin{array}{c}
r+3 / 4 \\
r+1
\end{array}\right)=\left(\frac{r^{2}+r+2 / 9}{r^{2}+r}\right) \frac{y_{r}}{r+1} .
$$


This implies

$$
y_{1}=3 / 16, \quad y_{r}=\frac{3}{16} \prod_{k=1}^{r-1} \frac{k^{2}+k+3 / 16}{k^{2}+k}
$$

Since

$$
\prod_{k=1}^{\infty} \frac{k^{2}+k+3 / 16}{k^{2}+k}=\frac{16}{3 \Gamma(1 / 4) \Gamma(3 / 4)}=\frac{16}{3 \sqrt{2} \pi},
$$

we obtain

$$
X_{r}<\frac{1}{\sqrt{2} \pi r}
$$

For $r \in \mathbb{N}$, we have

$$
\left(\begin{array}{c}
r-3 / 4 \\
r
\end{array}\right)>\left(\begin{array}{c}
r+1 / 4 \\
r+1
\end{array}\right)
$$

So when $g \in\{0,1\}$,

$$
\left(\begin{array}{c}
r-g+1 / 4 \\
r+1-g
\end{array}\right)\left(\begin{array}{c}
r-1 / 4 \\
r
\end{array}\right) \leq X_{r}
$$

which completes the proof.

10. Proof of the main theorems. Let us now assume that there are four distinct solutions $\left(x_{i}, y_{i}\right)$ to reduced form

$$
|F(x, y)| \leq h
$$

related to $\omega$ with $\left|y_{i}\right|>h^{3 / 4} /(3 I)^{1 / 8}$, corresponding to $\xi_{-1}, \xi_{0}, \xi_{1}$ and $\xi_{2}$, where we have ordered these in nondecreasing modulus. We will deduce a contradiction, implying that at most three such solutions can exist. Then Theorem 1.2 will be proven, since there are four choices of $\omega$.

We will show that $\left|\xi_{2}\right|$ is arbitrarily large in relation to $\left|\xi_{1}\right|$. By 29) and (30), we know that $\left|\xi_{1}\right|$ is large and hence $\left|\xi_{2}\right|$ is arbitrarily large, a contradiction.

Lemma 10.1. Let $F(x, y)$ be the quartic form. Suppose that $\left(x_{1}, y_{1}\right)$ and $\left(x_{2}, y_{2}\right)$ are two pairs of solutions to $|F(x, y)| \leq h$, both related to $\omega$, a fixed fourth root of unity. Put $\xi_{j}=\xi\left(x_{j}, y_{j}\right)$. Assume further that either

(i) $F(x, y)$ is the quartic form in Theorem 1.2 with

$$
4\left|A_{4}\right|^{1 / 8} h^{11 / 4} I^{9 / 8}<\left|\xi_{1}\right|<\left|\xi_{2}\right|,
$$

or

(ii) $F(x, y)$ is the quartic form in Theorem 1.1 with $I>135$ and

$$
0.39\left|A_{4}\right|^{1 / 8} h^{11 / 4} I^{9 / 8}<\left|\xi_{1}\right|<\left|\xi_{2}\right| .
$$


Then, for each positive integer $r$,

$$
\left|\xi_{2}\right|>\frac{4^{r} \sqrt{r}}{27} \frac{\left|A_{0}\right|^{1 / 8}}{\left(3\left|A_{4}\right|^{1 / 2}\right)^{1 / 2} h^{2 r+1}}\left(9 \sqrt{3 I\left|A_{4}\right|}\right)^{-2 r}\left|\xi_{1}\right|^{4 r+3} .
$$

Proof. We will use the upper bound (30) for case (i) and the upper bound (39) for case (ii). Note that (39) is a generalization for the upper bound (29) obtained to treat the equation $|F(x, y)|=1$. By (27),

$$
\left|\xi_{2}\right| \geq \frac{3\left|\xi_{1}\right|^{3}}{2 \pi h\left|A_{4}\right|^{1 / 4}} \text {. }
$$

This implies

$$
c_{1}(1,0)\left|\xi_{1}\right|^{5}\left|\xi_{2}\right|^{-3} \leq 4 h^{4} \pi \cdot 12^{3 / 2}\left(3 /\left|A_{0}\right|\right)^{1 / 2}\left|A_{4}\right|\left|\xi_{1}\right|^{-4} .
$$

Therefore, by $(30)$ or 39 and from the fact that $\left|A_{4}\right|<4 I$, we obtain

$$
c_{1}(1,0)\left|\xi_{1}\right|^{5}\left|\xi_{2}\right|^{-3}<0.01
$$

Lemma 8.1 implies that $\Sigma_{1,0} \neq 0$. So we may apply Lemma 9.1 to get

$$
c_{2}(1,0) I^{3}\left|\xi_{1}\right|^{-7}\left|\xi_{2}\right|>0.99 \text {. }
$$

One may now conclude

$$
\left|\xi_{2}\right|>\frac{0.99}{c_{2}(1,0)}\left|\xi_{1}\right|^{7}>0.93 h^{-3}\left(\frac{3\left|A_{4}\right|^{1 / 2}}{\left|A_{0}\right|}\right)^{-1 / 2}\left(9 \sqrt{3 I\left|A_{4}\right|}\right)^{-2}\left|\xi_{1}^{\prime}\right|^{7} .
$$

This proves the lemma for $r=1$. Moreover, we may conclude that

$$
c_{1}(2,0)\left|\xi_{1}\right|^{9}\left|\xi_{2}\right|^{-3}<\frac{18 h^{10} \sqrt{\pi} \cdot 16 \cdot(5 \cdot 27)^{3}\left|A_{4}\right|}{\left|A_{0}\right|^{2} 127^{3} \sqrt{2}}\left(9 \sqrt{3 I\left|A_{4}\right|}\right)^{6}\left|\xi_{1}\right|^{-12} .
$$

Since $\left|A_{4}\right| \leq 4 I$, by $(30)$ or $(39)$ we have

$$
c_{1}(2,0)\left|\xi_{1}\right|^{9}\left|\xi_{2}\right|^{-3}<0.1 \text {. }
$$

Via Lemmas 9.1 and 8.1 , we obtain

$$
\left|\xi_{2}\right|>\frac{0.9}{c_{2}(1,0)}\left|\xi_{1}\right|^{11}
$$

This yields the assertion of the lemma for $r=2$, after substituting the value of $c_{2}(2,0)$. To complete the proof, we use induction on $r$. Suppose that the assertion holds for some $r \geq 2$. Then

$$
c_{1}(r+1,0)\left|\xi_{1}\right|^{4 r+5}\left|\xi_{2}\right|^{-3}<\frac{18 \sqrt{\pi} \cdot 27^{3}\left|A_{4}\right| h^{6 r+4}}{\left|A_{0}\right|^{7 / 8} 4^{2 r-1} \sqrt{r+1} r \sqrt{r}}\left(9 \sqrt{3 I\left|A_{4}\right|}\right)^{6 r}\left|\xi_{1}\right|^{-8 r-4} .
$$

By (30) or (39), we have

$$
c_{1}(r+1,0)\left|\xi_{1}\right|^{4 r+5}\left|\xi_{2}\right|^{-3}<0.1 .
$$

If $\Sigma_{r+1,0} \neq 0$, then by Lemma 9.1 .

$$
c_{2}(r+1,0)\left|\xi_{1}\right|^{-4(r+1)-3}\left|\xi_{2}\right|>0.9 \text {. }
$$


Hence,

$$
\begin{aligned}
\left|\xi_{2}\right| & >\frac{0.9}{c_{2}(r+1,0)}\left|\xi_{1}\right|^{4(r+1)+3} \\
& >\frac{4^{r+1} \sqrt{r+1}}{27 h^{2 r+3}}\left(\frac{\left|A_{0}\right|}{3\left|A_{4}\right|^{1 / 2}}\right)^{1 / 2}(9 \sqrt{3 I})^{-2 r-2}\left|\xi_{1}\right|^{4 r+7} .
\end{aligned}
$$

If, however, $\Sigma_{r+1,0}=0$, then by Lemma 8.2, both $\Sigma_{r+1,1}$ and $\Sigma_{r+2,1}$ are nonzero and by Lemma 8.1, we have $r>5$. Using the induction hypothesis, we get

$$
c_{1}(r+1,1)\left|\xi_{1}\right|^{4 r+4}\left|\xi_{2}\right|^{-3}<0.01,
$$

and thus by Lemma 9.1, (10) and (29), we conclude

$$
c_{2}(r+1,1)\left|\xi_{1}\right|^{-4 r-4}\left|\xi_{2}\right|>0.99 .
$$

So, we obtain

$$
\left|\xi_{2}\right|>\frac{4^{r+1} \sqrt{r+1}\left|A_{0}\right|^{1 / 8}}{27 h^{2 r+2}\left|3 A_{4}\right|^{1 / 4}}\left(9 \sqrt{3 I\left|A_{4}\right|}\right)^{-2 r-1}\left|\xi_{1}\right|^{4(r+1)} .
$$

Consequently,

$$
\begin{aligned}
& c_{1}(r+2,1)\left|\xi_{1}\right|^{4 r+8}\left|\xi_{2}\right|^{-3} \\
& \qquad \frac{2 \sqrt{\pi} \cdot 27\left(3\left|A_{4}\right|\right)\left(9 \sqrt{3 I\left|A_{4}\right|}\right)^{6 r+3} h^{6 r+7}}{4^{2 r+1}(r+1) \sqrt{(r+1)(r+2)\left|A_{0}\right|}}\left|\xi_{1}\right|^{-8 r-4}<0.1 .
\end{aligned}
$$

A final application of Lemma 9.1 implies

$$
c_{2}(r+2,1)\left|\xi_{1}\right|^{-4 r-8}\left|\xi_{2}\right|>0.9,
$$

or

$$
\left|\xi_{2}\right|>\frac{0.9}{c_{2}(r+2,1)}\left|\xi_{1}\right|^{4 r+8}
$$

It follows that

$$
\left|\xi_{2}\right|>\frac{\sqrt{r+2} 4^{r+2}}{27} \frac{\left|A_{0}\right|^{1 / 8}}{3^{1 / 4} h^{2 r+4}}\left(9 \sqrt{3 I\left|A_{4}\right|}\right)^{-2 r-3}\left|\xi_{1}\right|^{4(r+1)+4} .
$$

Since $\left|\xi_{1}\right|>4 I^{9 / 8} h^{11 / 4}\left|A_{4}\right|^{1 / 8}$, we conclude that

$$
\left|\xi_{2}\right|>\frac{4^{r+1} \sqrt{r+1}}{27} \frac{\left|A_{0}\right|^{1 / 8}}{\left(3\left|A_{4}\right|^{1 / 2}\right)^{1 / 2}}\left(9 \sqrt{3 I\left|A_{4}\right|}\right)^{-2 r-2}\left|\xi_{1}\right|^{4 r+7}
$$

11. Forms with small discriminant. To finish the proof of Theorem 1.1. we need to study the quartic forms $F(x, y)=a_{0} x^{4}+a_{1} x^{3} y+$ $a_{2} x^{2} y^{2}+a_{3} x y^{3}+a_{4} y^{4}$ with $0<I_{F} \leq 135$ and $A_{0}=3\left(8 a_{0} a_{2}-3 a_{1}^{2}\right)<0$.

We followed an algorithm of Cremona, in Section 4.6 of [9], which gives all inequivalent integer quartics with given invariant $I$ and $J=0$. Using 
Magma, we counted the number of solutions to

$$
|F(x, y)|=1
$$

for all reduced quartic forms $F$ with $I_{F} \leq 135$ and $J_{F}=0$. Regarding $(x, y)$ and $(-x,-y)$ as the same, we did not find any form $F$ for which there are more than four solutions to $F(x, y)= \pm 1$. Our program was not efficient in the sense that it solves more than one equation from some equivalent classes. While reading the earlier versions of this paper, the referee has verified these computations in a very efficient way and kindly shared his results with the author. The following table contains all representatives of the complete set of binary forms $F$ with $I_{F} \leq 135$ and $J_{F}=0$ that split in $\mathbb{R}$.

\begin{tabular}{ll}
\hline$F(x, y)$ & $I_{F}$ \\
\hline$x^{4}-x^{3} y-6 x^{2} y^{2}+x y^{3}+y^{4}$ & 51 \\
$x^{4}+2 x^{3} y-6 x^{2} y^{2}-2 x y^{3}+y^{4}$ & 60 \\
$x^{4}-12 x^{2} y^{2}+16 x y^{3}-4 y^{4}$ & 96 \\
$x^{4}+8 x^{3} y+6 x^{2} y^{2}-4 x y^{3}-2 y^{4}$ & 108 \\
$x^{4}+x^{3} y-15 x^{2} y^{2}+18 x y^{3}-4 y^{4}$ & 123 \\
\hline
\end{tabular}

To solve the Thue equations $F(x, y)= \pm 1$ for forms $F$ in the above table, we may also use PARI since all of the binary forms in the table are monic. If

$$
F(x, y)=x^{4}-x^{3} y-6 x^{2} y^{2}+x y^{3}+y^{4}
$$

then $I_{F}=51$ and the solutions are

$$
(-1,0),(0,1),(1,2),(-2,1) .
$$

Note that we can write

$$
F(x, y)=x^{4}-x^{3} y-6 x^{2} y^{2}+x y^{3}+y^{4}=\xi(x, y)^{4}-\eta(x, y)^{4},
$$

so that $\eta(x, y) / \xi(x, y)=(x-i y) /(x+i y)$ and we have

$$
\frac{\eta(-1,0)}{\xi(-1,0)}=1, \quad \frac{\eta(0,1)}{\xi(0,1)}=-1, \quad \frac{\eta(1,2)}{\xi(1,2)}=-\frac{3+4 i}{5}, \quad \frac{\eta(-2,1)}{\xi(-2,1)}=\frac{3+4 i}{5} .
$$

This means $(-1,0)$ is related to $\omega=1,(0,1)$ is related to $\omega=-1,(1,2)$ is related to $\omega=-i$, and $(-2,1)$ is related to $\omega=i$. Therefore, one pair of solutions is related to each root of unity.

If

$$
F(x, y)=x^{4}+2 x^{3} y-6 x^{2} y^{2}-2 x y^{3}+y^{4}
$$

then $I_{F}=60$ and the solutions are $(1,0)$ and $(0,1)$.

If

$$
F(x, y)=x^{4}-12 x^{2} y^{2}+16 x y^{3}-4 y^{4}
$$


then $I_{F}=96$ and $F(x, y)=1$ has four solutions $(5,2),(1,3),(1,1),(1,0)$, while the equation $F(x, y)=-1$ has no solution.

If

$$
F(x, y)=x^{4}+8 x^{3} y+6 x^{2} y^{2}-4 x y^{3}-2 y^{4}
$$

then $I_{F}=108$ and the solutions are $(1,0)$ and $(-1,1)$.

If

$$
F(x, y)=x^{4}+x^{3} y-15 x^{2} y^{2}+18 x y^{3}-4 y^{4}
$$

then $I_{F}=123$ and the solutions are $(1,1)$ and $(1,0)$.

Acknowledgments. The author would like to thank Professor Michael Bennett for his support and insightful comments. The author is indebted to the anonymous referee for his very careful reading and valuable comments on the earlier version of this paper. The referee's suggestions certainly improved both presentation and mathematical contents of this manuscript.

\section{References}

[1] S. Akhtari, The Diophantine equation $a X^{4}-b Y^{2}=1$, J. Reine Angew. Math., to appear.

[2] S. Akhtari and R. Okazaki, The quartic Thue equations, J. Number Theory 130 (2010), 40-60.

[3] S. Akhtari, A. Togbé and P. G. Walsh, On the equation $a X^{4}-b Y^{2}=2$, Acta Arith. 131 (2008), 145-169.

[4] A. Baker, Contributions to the theory of Diophantine equations. I. On the representation of integers by binary forms, Philos. Trans. Roy. Soc. London Ser. A 263 (1967/1968), 173-191.

[5] - The theory of linear forms in logarithms, in: Transcendence Theory: Advances and Applications, A. Baker and D. W. Masser (eds.), Academic Press, 1977, 1-27.

[6] M. A. Bennett, On the representation of unity by binary cubic forms, Trans. Amer. Math. Soc. 353 (2001), 1507-1534.

[7] J. W. S. Cassels, An Introduction to the Geometry of Numbers, Springer, 1959.

[8] G. V. Chudnovsky, On the method of Thue-Siegel, Ann. of Math. 117 (1983), 325-382.

[9] J. E. Cremona, Reduction of binary cubic and quartic forms, LMS J. Comput. Math. 2 (1999), 64-94; Corrigendum, ibid. 4 (2001), 73.

[10] J. H. Evertse, On the equation $a x^{n}-b y^{n}=c$, Compos. Math. 47 (1982), 289-315.

[11] - On the representation of integers by binary cubic forms of positive discriminant, Invent. Math. 73 (1983), 117-138; Erratum, ibid. 75 (1984), 379.

[12] - Upper Bounds for the Numbers of Solutions of Diophantine Equations, Math. Centre Tracts 168, Mathematisch Centrum, Amsterdam, 1983.

[13] V. Krechmar, On the upper bound of the number of representations of an integer by binary forms of the fourth degree, Izv. Acad. Nauk SSSR Ser. Mat. 1939, 289-302 (in Russian).

[14] W. Ljunggren, On the representation of integers by certain binary cubic and biquadratic forms, Acta Arith. 17 (1971), 379-387. 
[15] C. L. Siegel, Über einige Anwendungen diophantischer Approximationen, Abh. Preuss. Akad. Wiss. 1929, no. 1, 70 pp.

[16] —, Einige Erläuterungen zu Thues Untersuchungen über Annäherungswerte algebraischer Zahlen und diophantische Gleichungen, Nachr. Akad. Wiss. Göttingen II Mat.-Phys. Kl. 1970, 169-195.

[17] -, Die Gleichung $a x^{n}-b y^{n}=c$, Math. Ann. 114 (1937), 57-68.

[18] K. Stromberg, An Introduction to Classical Real Analysis, Wadsworth, 1981.

[19] A. Thue, Bemerkungen über gewisse Näherungsbrüche algebraischer Zahlen, Christiania Vid.-Selsk. Skrifter 1908, no. 3, 34 pp.

[20] —, Über Annäherungenswerte algebraischen Zahlen, J. Reine Angew. Math. 135 (1909), 284-305.

[21] —, Über rationale Annäherungswerte der reellen Würzel der ganzen Funktion dritten Grades $x^{3}-a x-b$, Christiania Vid.-Selsk. Skrifter 1908, no. 6, 29 pp.

[22] —, Ein Fundamentaltheorem zur Bestimmung vou Annäherungswerten aller Würzeln gewisser ganzer Funktionen, J. Reine Angew. Math. 138 (1910), 96-108.

Shabnam Akhtari

Max-Planck-Institut für Mathematik

Vivatsgasse 7

53111 Bonn, Germany

E-mail: akhtari@mpim-bonn.mpg.de

Received on 18.6.2008

and in revised form on 27.4.2009 